\title{
Neoniphon pencei, a new species of holocentrid (Teleostei: Beryciformes) from Rarotonga, Cook Islands
}

\author{
Joshua M. Copus ${ }^{\dagger}$, Richard L. Pyle ${ }^{\ddagger}$, John L. Earle ${ }^{\ddagger}$ \\ † Hawaii Institute of Marine Biology, Kaneohe, United States of America \\ ‡ Bishop Museum, Honolulu, United States of America
}

Corresponding author: Joshua M. Copus (joshua.copus@gmail.com)

Academic editor: Rupert Collins

Received: 20 Oct 2014 | Accepted: 22 Jan 2015 | Published: 26 Jan 2015

Citation: Copus J, Pyle R, Earle J (2015) Neoniphon pencei, a new species of holocentrid (Teleostei:

Beryciformes) from Rarotonga, Cook Islands. Biodiversity Data Journal 3: e4180. doi: 10.3897/BDJ.3.e4180

\section{Abstract}

Neoniphon pencei, n. sp., is described from thirteen specimens, $132-197 \mathrm{~mm}$ standard length (SL) collected from mesophotic coral ecosystems (MCEs) at Rarotonga, Cook Islands by divers using mixed-gas closed-circuit rebreathers. It differs from all other species of the genus in number of lateral line scales, scales above and below lateral line, elements of life color, and in $\mathrm{COI}$ and cytochrome b DNA sequences. Of the five other known species of Neoniphon, it is most similar to the Indo-Pacific $N$. aurolineatus and the western Atlantic N. marianus both morphologically and genetically.

\section{Keywords}

Holocentridae, Neoniphon, new species, Mesophotic Coral Ecosystems, MCE, Rarotonga, Cook Islands 


\section{Introduction}

Collections of shore fishes at mesophotic depths ( $30-200 \mathrm{~m})$ across the Indo-Pacific are yielding a surprising number of undescribed species (Pyle 2000). Here, we describe a new species of the genus Neoniphon from depths of $90-115 \mathrm{~m}$ at Rarotonga, Cook Islands, raising to six the number of species within this genus.

The genus Neoniphon Castelnau 1875 includes five currently recognized species: $N$. argenteus (Valenciennes in Cuvier and Valenciennes 1831), N. aurolineatus (Liénard 1839), N. opercularis (Valenciennes in Cuvier and Valenciennes 1831) and N. sammara (Forsskål 1775) from the tropical Indo-Pacific, and $N$. marianus (Cuvier in Cuvier and Valenciennes 1829) from the tropical western Atlantic. Woods and Sonoda (1973) placed these five species (referring to $N$. argenteus by the junior synonym laevis [=læve] Günther 1859 , and to $N$. aurolineatus by the junior synonym scythrops Jordan and Evermann 1903) within the genus Flammeo Jordan and Evermann 1898, on the grounds that the earlier name Neoniphon (type species: N. armatus Castelnau 1875) was "based on a species whose status is uncertain" (p. 345). Randall and Heemstra (1985) treated the four IndoPacific species of the genus, and confidently asserted that the original description of $N$. armatus Castelnau 1875 (the type species ofNeoniphon) is conspecific with $N$. sammara, and therefore considered Neoniphon as a valid genus with higher nomenclatural priority, to apply to the the four Indo-Pacific species as well as $N$. marianus from the tropical Atlantic. The lead author is working on a larger phylogeographic study involving this genus, which will be published at a later time.

\section{Materials and methods}

Type specimens of the new species Neoniphon pencei from Rarotonga, Cook Islands have been deposited in the Bernice P. Bishop Museum, Honolulu (BPBM); the California Academy of Sciences, San Francisco (CAS); and the U.S. National Museum of Natural History, Washington, D.C. (USNM).

Measurements and counts given here follow the methods outlined in Randall (1998). Lengths of specimens are given as ratios of: standard length (SL) measured from the tip of the snout to the base of the caudal fin at the end of the hypural plate; body depth, taken at the point of maximum depth; or head length, measured from the median anterior point of the upper lip to the end of the longest opercular spine. Meristics and measurements were compared with data obtained from the literature for all five currently recognized species (Shimizu and Yamakawa 1979).

Tissue samples were obtained from each of the thirteen individuals of $N$. pencei collected at Rarotonga, Cook Islands by spear at 90-115 m. Tissue samples were also obtained from twenty-two specimens of the five other species of Neoniphon: $N$. sammara $(n=6)$ collected from Diego Garcia, British Indian Ocean Territory; $N$. opercularis $(n=2)$ collected from Moorea, French Polynesia; N. aurolineatus ( $n=7)$ collected from Oahu, Hawaii; N. marianus 
$(n=1)$ collected from the Commonwealth of the Bahamas; and $N$. argenteus $(n=6)$ collected from the Republic of Kiritimati. Total genomic DNA was extracted from each sample using the 'HotSHOT' protocol (Meeker et al. 2007). A 577-bp fragment of the mtDNA cytochrome $b(C y t b>)$ region was amplified using modified primers from Song et al. (1998) (5'TGAAGTTGTCGGGATCTCCT-3') and Taberlet et al. (1992) (5'TGCCGTGACGTAAACTATGG-3'). Polymerase chain reaction (PCR) was performed in a $15 \mu$ reaction containing $7.5 \mu$ BioMix Red (Biolone Inc., Springfield, NJ, USA), $0.2 \mu \mathrm{M}$ of each primer, 5-50 ng template DNA, and nanopure water (Thermo Scientific* Barnstead, Dubuque, IA, USA) to volume. PCR cycling parameters were as follows: initial $95^{\circ} \mathrm{C}$ denaturation for $10 \mathrm{~min}$. followed by 35 cycles of $94^{\circ} \mathrm{C}$ for $30 \mathrm{sec}, 60^{\circ} \mathrm{C}$ for $30 \mathrm{sec}$, and $72^{\circ}$ $\mathrm{C}$ for $30 \mathrm{sec}$, followed by a final extension of $72^{\circ} \mathrm{C}$ for $10 \mathrm{~min}$. PCR products were visualized using a 1.5\% agarose gel with GelStarTM (Cambrex Bio Science Rockland, Inc., Rockland MA, USA) and then cleaned by incubating with 0.75 units of Exonuclease and 0.5 units of Shrimp Akaline Phosphate (ExoSAP; USB, Cleveland, OH, USA) per $7.5 \mu \mathrm{l}$ of PCR product for $30 \mathrm{~min}$. at $37^{\circ} \mathrm{C}$ followed by $85^{\circ} \mathrm{C}$ for $15 \mathrm{~min}$. Sequencing was conducted in the forward direction and reverse direction when needed using a genetic analyzer (ABI 3130XL, Applied Biosystems, Foster City, California) at the Hawai'i Institute of Marine Biology EPSCoR Sequencing Facility. The sequences were aligned, edited and trimmed to a common length using Geneious Pro (v.5.6.6) DNA analysis software (Drummond et al. 2012). Twelve representative Cyt $b>$ haplotypes were deposited in GenBank (accession numbers KJ188431-188436 and KJ201921-201926). jModelTest v.2.1.4 (Darriba et al. 2012, Guindon and Gascuel 2003) was used with an Akaike information criterion (AIC) test to determine the best nucleotide substitution model for the data. The GTR+G model with gamma parameter 0.1840 was identified to be the best suited model for phylogenetic inference. Maximum Likelihood, Neighbor-Joining, and Maximum Parsimony tree-building methods were implemented using Mega v.5.2.2 (Tamura et al. 2011). Sargocentron rubrum (Genbank accession number AP004432.1) was used to root a maximum likelihood phylogenetic reconstruction. Clade support was evaluated by bootstrapping 1,000 replicates in all cases (Felsenstein 1985).

A DNA barcode (cytochrome c oxidase I; COI) was completed for the holotype and one paratype (BPBM 41196) using the primers from Baldwin et al. (2009), Fish-BCH (5'ACTTCYGGGTGRCCRAARAATCA-3') and Fish-BCL (5'TCAACYAATCAYAAAGATATYGGCAC-3') using the following PCR protocol: initial $95^{\circ} \mathrm{C}$ denaturation for $10 \mathrm{~min}$. followed by 35 cycles of $94^{\circ} \mathrm{C}$ for $30 \mathrm{sec}, 55^{\circ} \mathrm{C}$ for $30 \mathrm{sec}$, and $72^{\circ}$ $\mathrm{C}$ for $30 \mathrm{sec}$, followed by a final extension of $72^{\circ} \mathrm{C}$ for $10 \mathrm{~min}$. All other procedures were as described above. Both individuals possessed the same $\mathrm{COI}$ haplotype, so only one record was deposited in GenBank (http://www.ncbi.nlm.nih.gov/; accession number KJ188437) and BOLD (www.boldsystems.org; dx.doi.org/10.5883/DS-NPE511). 


\section{Taxon treatment}

\section{Neoniphon pencei Copus, Pyle, and Earle, sp. n.}

- ZooBank urn:Isid:zoobank.org:act:43F5CABA-6E4B-42BB-8569-8F93D3502DE9

- $\quad$ Barcode of Life NPE001-14

- GenBank KJ201926

- GenBank KJ188437

\section{Materials}

\section{Holotype:}

a. $\quad$ scientificName: Neoniphon pencei; originalNameUsage: Neoniphon pencei Copus, Pyle and Earle, 2014; originalNameUsagelD: 43f5caba-6e4b-42bb-8569-8f93d3502de9; namePublishedln: Copus, Joshua M., Richard L. Pyle \& John L. Earle. 2014. Neoniphon pencei, a new species of holocentrid from Rarotonga, Cook Islands. Biodiversity Data Journal.; namePublishedlnID: bbdce765-389b-4338-9c36-68def122f4fc; nameAccordingTo: Copus, Joshua M., Richard L. Pyle \& John L. Earle. 2014. Neoniphon pencei, a new species of holocentrid from Rarotonga, Cook Islands. Biodiversity Data Journal.; nameAccording TolD: bbdce765-389b-4338-9c36-68def122f4fc; acceptedNameUsage: Neoniphon pencei Copus, Pyle and Earle, 2014; acceptedNameUsage ID: 43f5caba-6e4b-42bb-8569-8f93d3502de9; taxonID: 43f5caba-6 e4b-42bb-8569-8f93d3502de9; scientificNamelD: 43f5caba-6e4b-42bb-8569-8f93d3502d e9; parentNameUsageID: b047f156-f8da-4ec6-9f64-87345b68a759; parentNameUsage: Neoniphon Castelnau, 1875; higherClassification: Animalia; Deuterostomia; Chordata; Craniata; Gnathostomata; Actinopterygii; Beryciformes; Holocentroidei; Holocentridae; Neoniphon; kingdom: Animalia; phylum: Chordata; class: Actinopterygii; order: Beryciformes; family: Holocentridae; taxonRank: species; vernacularName: Pence's Squirrelfish; nomenclaturalCode: ICZN; genus: Neoniphon; specificEpithet: pencei; scientificNameAuthorship: Copus, Pyle \& Earle; waterBody: Pacific Ocean; islandGroup: Cook Islands; island: Rarotonga; country: Cook Islands; countryCode: CK; locality: E side; Matavera; off Charles J. Boyle's house; verbatimLocality: Cook Islands; Rarotonga; E side; Matavera; off Charles J. Boyle's house; verbatimDepth: $115 \mathrm{~m}$; minimumDepthInMeters: 115; maximumDepthInMeters: 115; decimalLatitude: -21.223798; decimalLongitude: -159.728123; geodeticDatum: WGS 84; coordinateUncertaintyInMeters: 300; georeferenceSources: Google Earth; samplingProtocol: Spear; eventDate: 2012-07-02; year: 2012; month: 7; day: 2; habitat: small cave near base of vertical drop-off; individualCount: 1; lifeStage: adult; preparations: 55\% Isopropyl; catalogNumber: 41197; recordedBy: David F. Pence; disposition: in collection; associatedSequences: GenBank KJ201926 (cyt b); KJ188437 (COI); identifiedBy: Richard L. Pyle; dateldentified: 2012-08-07; modified: 2014-10-09T23:30:00Z; language: en; collectionID: urn:Isid:biocol.org:col:1001; institutionCode: BPBM; collectionCode: Fish; basisOfRecord: PreservedSpecimen

\section{Paratypes:}

a. $\quad$ scientificName: Neoniphon pencei; originalNameUsage: Neoniphon pencei Copus, Pyle and Earle, 2014; originalNameUsagelD: 43f5caba-6e4b-42bb-8569-8f93d3502de9; namePublishedln: Copus, Joshua M., Richard L. Pyle \& John L. Earle. 2014. Neoniphon pencei, a new species of holocentrid from Rarotonga, Cook Islands. Biodiversity Data Journal.; namePublishedlnID: bbdce765-389b-4338-9c36-68def122f4fc; 
nameAccordingTo: Copus, Joshua M., Richard L. Pyle \& John L. Earle. 2014. Neoniphon pencei, a new species of holocentrid from Rarotonga, Cook Islands. Biodiversity Data Journal.; nameAccordingToID: bbdce765-389b-4338-9c36-68def122f4fc; acceptedNameUsage: Neoniphon pencei Copus, Pyle and Earle, 2014; acceptedNameUsage ID: 43f5caba-6e4b-42bb-8569-8f93d3502de9; taxonID: $\underline{43 f 5 c a b a-6}$ e4b-42bb-8569-8f93d3502de9; scientificNamelD: 43f5caba-6e4b-42bb-8569-8f93d3502d e9; parentNameUsagelD: b047f156-f8da-4ec6-9f64-87345b68a759; parentNameUsage: Neoniphon Castelnau, 1875; higherClassification: Animalia; Deuterostomia; Chordata; Craniata; Gnathostomata; Actinopterygii; Beryciformes; Holocentroidei; Holocentridae; Neoniphon; kingdom: Animalia; phylum: Chordata; class: Actinopterygii; order: Beryciformes; family: Holocentridae; taxonRank: species; vernacularName: Pence's Squirrelfish; nomenclaturalCode: ICZN; genus: Neoniphon; specificEpithet: pencei; scientificNameAuthorship: Copus, Pyle \& Earle; waterBody: Pacific Ocean; islandGroup: Cook Islands; island: Rarotonga; country: Cook Islands; countryCode: CK; locality: E side; Matavera; off Charles J. Boyle's house; verbatimLocality: Cook Islands; Rarotonga; E side; Matavera; off Charles J. Boyle's house; verbatimDepth: $115 \mathrm{~m}$; minimumDepthInMeters: 115; maximumDepthInMeters: 115; decimalLatitude: -21.223798; decimalLongitude: -159.728123; geodeticDatum: WGS 84; coordinateUncertaintyInMeters: 300; georeferenceSources: Google Earth; samplingProtocol: Spear; eventDate: 2012-07-03; year: 2012; month: 7; day: 3; habitat: small cave near base of vertical drop-off; individualCount: 1; lifeStage: adult; preparations: 55\% Isopropyl; catalogNumber: 41196; recordedBy: David F. Pence; disposition: in collection; associatedSequences: GenBank KJ201926; identifiedBy: Richard L. Pyle; dateldentified: 2012-08-07; modified: 2014-10-09T23:30:00Z; language: en; collectionID: urn:Isid:biocol.org:col:1001; institutionCode: BPBM; collectionCode: Fish; basisOfRecord: PreservedSpecimen

b. ScientificName: Neoniphon pencei; originalNameUsage: Neoniphon pencei Copus, Pyle and Earle, 2014; originalNameUsagelD: 43f5caba-6e4b-42bb-8569-8f93d3502de9; namePublishedln: Copus, Joshua M., Richard L. Pyle \& John L. Earle. 2014. Neoniphon pencei, a new species of holocentrid from Rarotonga, Cook Islands. Biodiversity Data Journal.; namePublishedInID: bbdce765-389b-4338-9c36-68def122f4fc; nameAccordingTo: Copus, Joshua M., Richard L. Pyle \& John L. Earle. 2014. Neoniphon pencei, a new species of holocentrid from Rarotonga, Cook Islands. Biodiversity Data Journal.; nameAccording ToID: bbdce765-389b-4338-9c36-68def122f4fc; acceptedNameUsage: Neoniphon pencei Copus, Pyle and Earle, 2014; acceptedNameUsage ID: 43f5caba-6e4b-42bb-8569-8f93d3502de9; taxonID: 43f5caba-6 e4b-42bb-8569-8f93d3502de9; scientificNamelD: 43f5caba-6e4b-42bb-8569-8f93d3502d e9; parentNameUsageID: b047f156-f8da-4ec6-9f64-87345b68a759; parentNameUsage: Neoniphon Castelnau, 1875; higherClassification: Animalia; Deuterostomia; Chordata; Craniata; Gnathostomata; Actinopterygii; Beryciformes; Holocentroidei; Holocentridae; Neoniphon; kingdom: Animalia; phylum: Chordata; class: Actinopterygii; order: Beryciformes; family: Holocentridae; taxonRank: species; vernacularName: Pence's Squirrelfish; nomenclaturalCode: ICZN; genus: Neoniphon; specificEpithet: pencei; scientificNameAuthorship: Copus, Pyle \& Earle; waterBody: Pacific Ocean; islandGroup: Cook Islands; island: Rarotonga; country: Cook Islands; countryCode: CK; locality: E side; Matavera; off Charles J. Boyle's house; verbatimLocality: Cook Islands; Rarotonga; E side; Matavera; off Charles J. Boyle's house; verbatimDepth: $115 \mathrm{~m}$; minimumDepthInMeters: 115; maximumDepthInMeters: 115; decimalLatitude: -21.223798; decimalLongitude: -159.728123; geodeticDatum: WGS 84; coordinateUncertaintyInMeters: 300; georeferenceSources: Google Earth; 
samplingProtocol: Spear; eventDate: 2012-07-03; year: 2012; month: 7; day: 3; habitat: small cave near base of vertical drop-off; individualCount: 1; lifeStage: adult; preparations: 55\% Isopropyl; catalogNumber: 41196; recordedBy: David F. Pence; disposition: in collection; associatedSequences: GenBank KJ201926; identifiedBy: Richard L. Pyle; dateldentified: 2012-08-07; modified: 2014-10-09T23:30:00Z; language: en; collectionID: urn:Isid:biocol.org:col:1001; institutionCode: BPBM; collectionCode: Fish; basisOfRecord: PreservedSpecimen

c. ScientificName: Neoniphon pencei; originalNameUsage: Neoniphon pencei Copus, Pyle and Earle, 2014; originalNameUsagelD: 43f5caba-6e4b-42bb-8569-8f93d3502de9; namePublishedln: Copus, Joshua M., Richard L. Pyle \& John L. Earle. 2014. Neoniphon pencei, a new species of holocentrid from Rarotonga, Cook Islands. Biodiversity Data Journal.; namePublishedlnID: bbdce765-389b-4338-9c36-68def122f4fc; nameAccordingTo: Copus, Joshua M., Richard L. Pyle \& John L. Earle. 2014. Neoniphon pencei, a new species of holocentrid from Rarotonga, Cook Islands. Biodiversity Data Journal.; nameAccordingToID: bbdce765-389b-4338-9c36-68def122f4fc; acceptedNameUsage: Neoniphon pencei Copus, Pyle and Earle, 2014; acceptedNameUsageID: 43f5caba-6e4b-42bb-8569-8f93d3502de9; taxonID: 43f5caba-6 e4b-42bb-8569-8f93d3502de9; scientificNamelD: 43f5caba-6e4b-42bb-8569-8f93d3502d e9; parentNameUsageID: b047f156-f8da-4ec6-9f64-87345b68a759; parentNameUsage: Neoniphon Castelnau, 1875; higherClassification: Animalia; Deuterostomia; Chordata; Craniata; Gnathostomata; Actinopterygii; Beryciformes; Holocentroidei; Holocentridae; Neoniphon; kingdom: Animalia; phylum: Chordata; class: Actinopterygii; order: Beryciformes; family: Holocentridae; taxonRank: species; vernacularName: Pence's Squirrelfish; nomenclaturalCode: ICZN; genus: Neoniphon; specificEpithet: pencei; scientificNameAuthorship: Copus, Pyle \& Earle; waterBody: Pacific Ocean; islandGroup: Cook Islands; island: Rarotonga; country: Cook Islands; countryCode: CK; locality: E side; Matavera; off Charles J. Boyle's house; verbatimLocality: Cook Islands; Rarotonga; E side; Matavera; off Charles J. Boyle's house; verbatimDepth: 115 m; minimumDepthInMeters: 115; maximumDepthInMeters: 115; decimalLatitude: -21.223798; decimalLongitude: -159.728123; geodeticDatum: WGS 84; coordinateUncertaintyInMeters: 300; georeferenceSources: Google Earth; samplingProtocol: Spear; eventDate: 2012-07-03; year: 2012; month: 7; day: 3; habitat: small cave near base of vertical drop-off; individualCount: 1; lifeStage: adult; preparations: 55\% Isopropyl; catalogNumber: 41196; recordedBy: David F. Pence; disposition: in collection; associatedSequences: GenBank KJ201926; identifiedBy: Richard L. Pyle; dateldentified: 2012-08-07; modified: 2014-10-09T23:30:00Z; language: en; collectionID: urn:Isid:biocol.org:col:1001; institutionCode: BPBM; collectionCode: Fish; basisOfRecord: PreservedSpecimen

d. ScientificName: Neoniphon pencei; originalNameUsage: Neoniphon pencei Copus, Pyle and Earle, 2014; originalNameUsagelD: 43f5caba-6e4b-42bb-8569-8f93d3502de9; namePublishedln: Copus, Joshua M., Richard L. Pyle \& John L. Earle. 2014. Neoniphon pencei, a new species of holocentrid from Rarotonga, Cook Islands. Biodiversity Data Journal.; namePublishedlnID: bbdce765-389b-4338-9c36-68def122f4fc; nameAccordingTo: Copus, Joshua M., Richard L. Pyle \& John L. Earle. 2014. Neoniphon pencei, a new species of holocentrid from Rarotonga, Cook Islands. Biodiversity Data Journal.; nameAccording TolD: bbdce765-389b-4338-9c36-68def122f4fc; acceptedNameUsage: Neoniphon pencei Copus, Pyle and Earle, 2014; acceptedNameUsageID: 43f5caba-6e4b-42bb-8569-8f93d3502de9; taxonID: 43f5caba-6 e4b-42bb-8569-8f93d3502de9; scientificNamelD: 43f5caba-6e4b-42bb-8569-8f93d3502d e9; parentNameUsageID: b047f156-f8da-4ec6-9f64-87345b68a759; parentNameUsage: 
Neoniphon Castelnau, 1875; higherClassification: Animalia; Deuterostomia; Chordata; Craniata; Gnathostomata; Actinopterygii; Beryciformes; Holocentroidei; Holocentridae; Neoniphon; kingdom: Animalia; phylum: Chordata; class: Actinopterygii; order: Beryciformes; family: Holocentridae; taxonRank: species; vernacularName: Pence's Squirrelfish; nomenclaturalCode: ICZN; genus: Neoniphon; specificEpithet: pencei; scientificNameAuthorship: Copus, Pyle \& Earle; waterBody: Pacific Ocean; islandGroup: Cook Islands; island: Rarotonga; country: Cook Islands; countryCode: CK; locality: E side; Matavera; off Charles J. Boyle's house; verbatimLocality: Cook Islands; Rarotonga; E side; Matavera; off Charles J. Boyle's house; verbatimDepth: $115 \mathrm{~m}$; minimumDepthInMeters: 115; maximumDepthInMeters: 115; decimalLatitude: -21.223798; decimalLongitude: -159.728123; geodeticDatum: WGS 84; coordinateUncertaintyInMeters: 300; georeferenceSources: Google Earth; samplingProtocol: Spear; eventDate: 2012-07-03; year: 2012; month: 7; day: 3; habitat: small cave near base of vertical drop-off; individualCount: 1; lifeStage: adult; preparations: 55\% Isopropyl; catalogNumber: 41196; recordedBy: David F. Pence; disposition: in collection; associatedSequences: GenBank KJ201926; identifiedBy: Richard L. Pyle; dateldentified: 2012-08-07; modified: 2014-10-09T23:30:00Z; language: en; collectionID: urn:Isid:biocol.org:col:1001; institutionCode: BPBM; collectionCode: Fish; basisOfRecord: PreservedSpecimen

e. ScientificName: Neoniphon pencei; originalNameUsage: Neoniphon pencei Copus, Pyle and Earle, 2014; originalNameUsageID: 43f5caba-6e4b-42bb-8569-8f93d3502de9; namePublishedln: Copus, Joshua M., Richard L. Pyle \& John L. Earle. 2014. Neoniphon pencei, a new species of holocentrid from Rarotonga, Cook Islands. Biodiversity Data Journal.; namePublishedInID: bbdce765-389b-4338-9c36-68def122f4fc; nameAccordingTo: Copus, Joshua M., Richard L. Pyle \& John L. Earle. 2014. Neoniphon pencei, a new species of holocentrid from Rarotonga, Cook Islands. Biodiversity Data Journal.; nameAccordingToID: bbdce765-389b-4338-9c36-68def122f4fc; acceptedNameUsage: Neoniphon pencei Copus, Pyle and Earle, 2014; acceptedNameUsage ID: 43f5caba-6e4b-42bb-8569-8f93d3502de9; taxonID: 43f5caba-6 e4b-42bb-8569-8f93d3502de9; scientificNamelD: 43f5caba-6e4b-42bb-8569-8f93d3502d e9; parentNameUsagelD: b047f156-f8da-4ec6-9f64-87345b68a759; parentNameUsage: Neoniphon Castelnau, 1875; higherClassification: Animalia; Deuterostomia; Chordata; Craniata; Gnathostomata; Actinopterygii; Beryciformes; Holocentroidei; Holocentridae; Neoniphon; kingdom: Animalia; phylum: Chordata; class: Actinopterygii; order: Beryciformes; family: Holocentridae; taxonRank: species; vernacularName: Pence's Squirrelfish; nomenclaturalCode: ICZN; genus: Neoniphon; specificEpithet: pencei; scientificNameAuthorship: Copus, Pyle \& Earle; waterBody: Pacific Ocean; islandGroup: Cook Islands; island: Rarotonga; country: Cook Islands; countryCode: CK; locality: E side; Matavera; off Charles J. Boyle's house; verbatimLocality: Cook Islands; Rarotonga; E side; Matavera; off Charles J. Boyle's house; verbatimDepth: $115 \mathrm{~m}$; minimumDepthInMeters: 115; maximumDepthInMeters: 115; decimalLatitude: -21.223798; decimalLongitude: -159.728123; geodeticDatum: WGS 84; coordinateUncertaintyInMeters: 300; georeferenceSources: Google Earth; samplingProtocol: Spear; eventDate: 2012-07-03; year: 2012; month: 7; day: 3; habitat: small cave near base of vertical drop-off; individualCount: 1; lifeStage: adult; preparations: 55\% Isopropyl; catalogNumber: 41196; recordedBy: David F. Pence; disposition: in collection; associatedSequences: GenBank KJ201926; identifiedBy: Richard L. Pyle; dateldentified: 2012-08-07; modified: 2014-10-09T23:30:00Z; language: en; collectionID: urn:Isid:biocol.org:col:1001; institutionCode: BPBM; collectionCode: Fish; basisOfRecord: PreservedSpecimen 
f. ScientificName: Neoniphon pencei; originalNameUsage: Neoniphon pencei Copus, Pyle and Earle, 2014; originalNameUsagelD: 43f5caba-6e4b-42bb-8569-8f93d3502de9; namePublishedln: Copus, Joshua M., Richard L. Pyle \& John L. Earle. 2014. Neoniphon pencei, a new species of holocentrid from Rarotonga, Cook Islands. Biodiversity Data Journal.; namePublishedInID: bbdce765-389b-4338-9c36-68def122f4fc; nameAccordingTo: Copus, Joshua M., Richard L. Pyle \& John L. Earle. 2014. Neoniphon pencei, a new species of holocentrid from Rarotonga, Cook Islands. Biodiversity Data Journal.; nameAccordingToID: bbdce765-389b-4338-9c36-68def122f4fc; acceptedNameUsage: Neoniphon pencei Copus, Pyle and Earle, 2014; acceptedNameUsage ID: 43f5caba-6e4b-42bb-8569-8f93d3502de9; taxonID: 43f5caba-6 e4b-42bb-8569-8f93d3502de9; scientificNamelD: 43f5caba-6e4b-42bb-8569-8f93d3502d e9; parentNameUsageID: b047f156-f8da-4ec6-9f64-87345b68a759; parentNameUsage: Neoniphon Castelnau, 1875; higherClassification: Animalia; Deuterostomia; Chordata; Craniata; Gnathostomata; Actinopterygii; Beryciformes; Holocentroidei; Holocentridae; Neoniphon; kingdom: Animalia; phylum: Chordata; class: Actinopterygii; order: Beryciformes; family: Holocentridae; taxonRank: species; vernacularName: Pence's Squirrelfish; nomenclaturalCode: ICZN; genus: Neoniphon; specificEpithet: pencei; scientificNameAuthorship: Copus, Pyle \& Earle; waterBody: Pacific Ocean; islandGroup: Cook Islands; island: Rarotonga; country: Cook Islands; countryCode: CK; locality: E side; Matavera; off Charles J. Boyle's house; verbatimLocality: Cook Islands; Rarotonga; E side; Matavera; off Charles J. Boyle's house; verbatimDepth: $115 \mathrm{~m}$; minimumDepthInMeters: 115; maximumDepthInMeters: 115; decimalLatitude: -21.223798; decimalLongitude: -159.728123; geodeticDatum: WGS 84; coordinateUncertaintyInMeters: 300; georeferenceSources: Google Earth; samplingProtocol: Spear; eventDate: 2012-07-03; year: 2012; month: 7; day: 3; habitat: small cave near base of vertical drop-off; individualCount: 1; lifeStage: adult; preparations: 55\% Isopropyl; catalogNumber: 41196; recordedBy: David F. Pence; disposition: in collection; associatedSequences: GenBank KJ201926; identifiedBy: Richard L. Pyle; dateldentified: 2012-08-07; modified: 2014-10-09T23:30:00Z; language: en; collectionID: urn:Isid:biocol.org:col:1001; institutionCode: BPBM; collectionCode: Fish; basisOfRecord: PreservedSpecimen

g. ScientificName: Neoniphon pencei; originalNameUsage: Neoniphon pencei Copus, Pyle and Earle, 2014; originalNameUsagelD: 43f5caba-6e4b-42bb-8569-8f93d3502de9; namePublishedln: Copus, Joshua M., Richard L. Pyle \& John L. Earle. 2014. Neoniphon pencei, a new species of holocentrid from Rarotonga, Cook Islands. Biodiversity Data Journal.; namePublishedInID: bbdce765-389b-4338-9c36-68def122f4fc; nameAccordingTo: Copus, Joshua M., Richard L. Pyle \& John L. Earle. 2014. Neoniphon pencei, a new species of holocentrid from Rarotonga, Cook Islands. Biodiversity Data Journal.; nameAccording TolD: bbdce765-389b-4338-9c36-68def122f4fc; acceptedNameUsage: Neoniphon pencei Copus, Pyle and Earle, 2014; acceptedNameUsageID: 43f5caba-6e4b-42bb-8569-8f93d3502de9; taxonID: 43f5caba-6 e4b-42bb-8569-8f93d3502de9; scientificNamelD: 43f5caba-6e4b-42bb-8569-8f93d3502d e9; parentNameUsageID: b047f156-f8da-4ec6-9f64-87345b68a759; parentNameUsage: Neoniphon Castelnau, 1875; higherClassification: Animalia; Deuterostomia; Chordata; Craniata; Gnathostomata; Actinopterygii; Beryciformes; Holocentroidei; Holocentridae; Neoniphon; kingdom: Animalia; phylum: Chordata; class: Actinopterygii; order: Beryciformes; family: Holocentridae; taxonRank: species; vernacularName: Pence's Squirrelfish; nomenclaturalCode: ICZN; genus: Neoniphon; specificEpithet: pencei; scientificNameAuthorship: Copus, Pyle \& Earle; waterBody: Pacific Ocean; islandGroup: Cook Islands; island: Rarotonga; country: Cook Islands; countryCode: CK; locality: E side; 
Matavera; off Charles J. Boyle's house; verbatimLocality: Cook Islands; Rarotonga; E side; Matavera; off Charles J. Boyle's house; verbatimDepth: $115 \mathrm{~m}$; minimumDepthInMeters: 115; maximumDepthInMeters: 115; decimalLatitude: -21.223798; decimalLongitude: -159.728123; geodeticDatum: WGS 84; coordinateUncertaintyInMeters: 300; georeferenceSources: Google Earth; samplingProtocol: Spear; eventDate: 2012-07-03; year: 2012; month: 7; day: 3; habitat: small cave near base of vertical drop-off; individualCount: 1; lifeStage: adult; preparations: 55\% Isopropyl; catalogNumber: 41196; recordedBy: David F. Pence; disposition: in collection; associatedSequences: GenBank KJ201926; identifiedBy: Richard L. Pyle; dateldentified: 2012-08-07; modified: 2014-10-09T23:30:00Z; language: en; collectionID: urn:Isid:biocol.org:col:1001; institutionCode: BPBM; collectionCode: Fish; basisOfRecord: PreservedSpecimen

h. ScientificName: Neoniphon pencei; originalNameUsage: Neoniphon pencei Copus, Pyle and Earle, 2014; originalNameUsagelD: 43f5caba-6e4b-42bb-8569-8f93d3502de9; namePublishedln: Copus, Joshua M., Richard L. Pyle \& John L. Earle. 2014. Neoniphon pencei, a new species of holocentrid from Rarotonga, Cook Islands. Biodiversity Data Journal.; namePublishedInID: bbdce765-389b-4338-9c36-68def122f4fc; nameAccordingTo: Copus, Joshua M., Richard L. Pyle \& John L. Earle. 2014. Neoniphon pencei, a new species of holocentrid from Rarotonga, Cook Islands. Biodiversity Data Journal.; nameAccording TolD: bbdce765-389b-4338-9c36-68def122f4fc; acceptedNameUsage: Neoniphon pencei Copus, Pyle and Earle, 2014; acceptedNameUsage ID: 43f5caba-6e4b-42bb-8569-8f93d3502de9; taxonID: $\underline{43 f 5 c a b a-6}$ e4b-42bb-8569-8f93d3502de9; scientificNameID: 43f5caba-6e4b-42bb-8569-8f93d3502d e9; parentNameUsageID: b047f156-f8da-4ec6-9f64-87345b68a759; parentNameUsage: Neoniphon Castelnau, 1875; higherClassification: Animalia; Deuterostomia; Chordata; Craniata; Gnathostomata; Actinopterygii; Beryciformes; Holocentroidei; Holocentridae; Neoniphon; kingdom: Animalia; phylum: Chordata; class: Actinopterygii; order: Beryciformes; family: Holocentridae; taxonRank: species; vernacularName: Pence's Squirrelfish; nomenclaturalCode: ICZN; genus: Neoniphon; specificEpithet: pencei; scientificNameAuthorship: Copus, Pyle \& Earle; waterBody: Pacific Ocean; islandGroup: Cook Islands; island: Rarotonga; country: Cook Islands; countryCode: CK; locality: E side; Matavera; off Charles J. Boyle's house; verbatimLocality: Cook Islands; Rarotonga; E side; Matavera; off Charles J. Boyle's house; verbatimDepth: 115 m; minimumDepthInMeters: 115; maximumDepthInMeters: 115; decimalLatitude: -21.223798; decimalLongitude: -159.728123; geodeticDatum: WGS 84; coordinateUncertaintyInMeters: 300; georeferenceSources: Google Earth; samplingProtocol: Spear; eventDate: 2012-07-03; year: 2012; month: 7; day: 3; habitat: small cave near base of vertical drop-off; individualCount: 1; lifeStage: adult; preparations: 55\% Isopropyl; catalogNumber: 41196; recordedBy: David F. Pence; disposition: in collection; associatedSequences: GenBank KJ201926; identifiedBy: Richard L. Pyle; dateldentified: 2012-08-07; modified: 2014-10-09T23:30:00Z; language: en; collectionID: urn:Isid:biocol.org:col:1001; institutionCode: BPBM; collectionCode: Fish; basisOfRecord: PreservedSpecimen

i. ScientificName: Neoniphon pencei; originalNameUsage: Neoniphon pencei Copus, Pyle and Earle, 2014; originalNameUsagelD: 43f5caba-6e4b-42bb-8569-8f93d3502de9; namePublishedln: Copus, Joshua M., Richard L. Pyle \& John L. Earle. 2014. Neoniphon pencei, a new species of holocentrid from Rarotonga, Cook Islands. Biodiversity Data Journal.; namePublishedlnID: bbdce765-389b-4338-9c36-68def122f4fc; nameAccordingTo: Copus, Joshua M., Richard L. Pyle \& John L. Earle. 2014. Neoniphon pencei, a new species of holocentrid from Rarotonga, Cook Islands. Biodiversity Data 
Journal.; nameAccordingTolD: bbdce765-389b-4338-9c36-68def122f4fc; acceptedNameUsage: Neoniphon pencei Copus, Pyle and Earle, 2014; acceptedNameUsage ID: 43f5caba-6e4b-42bb-8569-8f93d3502de9; taxonID: 43f5caba-6 e4b-42bb-8569-8f93d3502de9; scientificNamelD: 43f5caba-6e4b-42bb-8569-8f93d3502d e9; parentNameUsageID: b047f156-f8da-4ec6-9f64-87345b68a759; parentNameUsage: Neoniphon Castelnau, 1875; higherClassification: Animalia; Deuterostomia; Chordata; Craniata; Gnathostomata; Actinopterygii; Beryciformes; Holocentroidei; Holocentridae; Neoniphon; kingdom: Animalia; phylum: Chordata; class: Actinopterygii; order: Beryciformes; family: Holocentridae; taxonRank: species; vernacularName: Pence's Squirrelfish; nomenclaturalCode: ICZN; genus: Neoniphon; specificEpithet: pencei; scientificNameAuthorship: Copus, Pyle \& Earle; waterBody: Pacific Ocean; islandGroup: Cook Islands; island: Rarotonga; country: Cook Islands; countryCode: CK; locality: E side; Matavera; off Charles J. Boyle's house; verbatimLocality: Cook Islands; Rarotonga; E side; Matavera; off Charles J. Boyle's house; verbatimDepth: 115 m; minimumDepthInMeters: 115; maximumDepthInMeters: 115; decimalLatitude: -21.223798; decimalLongitude: -159.728123; geodeticDatum: WGS 84; coordinateUncertaintyInMeters: 300; georeferenceSources: Google Earth; samplingProtocol: Spear; eventDate: 2012-07-03; year: 2012; month: 7; day: 3; habitat: small cave near base of vertical drop-off; individualCount: 1; lifeStage: adult; preparations: 55\% Isopropyl; catalogNumber: 41196; recordedBy: David F. Pence; disposition: in collection; associatedSequences: GenBank KJ201926; identifiedBy: Richard L. Pyle; dateldentified: 2012-08-07; modified: 2014-10-09T23:30:00Z; language: en; collectionID: urn:Isid:biocol.org:col:1001; institutionCode: BPBM; collectionCode: Fish; basisOfRecord: PreservedSpecimen

j. $\quad$ scientificName: Neoniphon pencei; originalNameUsage: Neoniphon pencei Copus, Pyle and Earle, 2014; originalNameUsagelD: 43f5caba-6e4b-42bb-8569-8f93d3502de9; namePublishedln: Copus, Joshua M., Richard L. Pyle \& John L. Earle. 2014. Neoniphon pencei, a new species of holocentrid from Rarotonga, Cook Islands. Biodiversity Data Journal.; namePublishedlnID: bbdce765-389b-4338-9c36-68def122f4fc; nameAccordingTo: Copus, Joshua M., Richard L. Pyle \& John L. Earle. 2014. Neoniphon pencei, a new species of holocentrid from Rarotonga, Cook Islands. Biodiversity Data Journal.; nameAccordingToID: bbdce765-389b-4338-9c36-68def122f4fc; acceptedNameUsage: Neoniphon pencei Copus, Pyle and Earle, 2014; acceptedNameUsage ID: 43f5caba-6e4b-42bb-8569-8f93d3502de9; taxonID: 43f5caba-6 e4b-42bb-8569-8f93d3502de9; scientificNameID: 43f5caba-6e4b-42bb-8569-8f93d3502d e9; parentNameUsageID: b047f156-f8da-4ec6-9f64-87345b68a759; parentNameUsage: Neoniphon Castelnau, 1875; higherClassification: Animalia; Deuterostomia; Chordata; Craniata; Gnathostomata; Actinopterygii; Beryciformes; Holocentroidei; Holocentridae; Neoniphon; kingdom: Animalia; phylum: Chordata; class: Actinopterygii; order: Beryciformes; family: Holocentridae; taxonRank: species; vernacularName: Pence's Squirrelfish; nomenclaturalCode: ICZN; genus: Neoniphon; specificEpithet: pencei; scientificNameAuthorship: Copus, Pyle \& Earle; waterBody: Pacific Ocean; islandGroup: Cook Islands; island: Rarotonga; country: Cook Islands; countryCode: CK; locality: E side; Matavera; off Charles J. Boyle's house; verbatimLocality: Cook Islands; Rarotonga; E side; Matavera; off Charles J. Boyle's house; verbatimDepth: $115 \mathrm{~m}$; minimumDepthInMeters: 115; maximumDepthInMeters: 115; decimalLatitude: -21.223798; decimalLongitude: -159.728123; geodeticDatum: WGS 84; coordinateUncertaintyInMeters: 300; georeferenceSources: Google Earth; samplingProtocol: Spear; eventDate: 2012-07-03; year: 2012; month: 7; day: 3; habitat: small cave near base of vertical drop-off; individualCount: 1; lifeStage: adult; preparations: 
55\% Isopropyl; catalogNumber: 41196; recordedBy: David F. Pence; disposition: in collection; associatedSequences: GenBank KJ201926; identifiedBy: Richard L. Pyle; dateldentified: 2012-08-07; modified: 2014-10-09T23:30:00Z; language: en; collectionID: urn:Isid:biocol.org:col:1001; institutionCode: BPBM; collectionCode: Fish; basisOfRecord: PreservedSpecimen

k. ScientificName: Neoniphon pencei; originalNameUsage: Neoniphon pencei Copus, Pyle and Earle, 2014; originalNameUsagelD: 43f5caba-6e4b-42bb-8569-8f93d3502de9; namePublishedln: Copus, Joshua M., Richard L. Pyle \& John L. Earle. 2014. Neoniphon pencei, a new species of holocentrid from Rarotonga, Cook Islands. Biodiversity Data Journal.; namePublishedInID: bbdce765-389b-4338-9c36-68def122f4fc; nameAccordingTo: Copus, Joshua M., Richard L. Pyle \& John L. Earle. 2014. Neoniphon pencei, a new species of holocentrid from Rarotonga, Cook Islands. Biodiversity Data Journal.; nameAccording ToID: bbdce765-389b-4338-9c36-68def122f4fc; acceptedNameUsage: Neoniphon pencei Copus, Pyle and Earle, 2014; acceptedNameUsageID: 43f5caba-6e4b-42bb-8569-8f93d3502de9; taxonID: 43f5caba-6 e4b-42bb-8569-8f93d3502de9; scientificNamelD: 43f5caba-6e4b-42bb-8569-8f93d3502d e9; parentNameUsagelD: b047f156-f8da-4ec6-9f64-87345b68a759; parentNameUsage: Neoniphon Castelnau, 1875; higherClassification: Animalia; Deuterostomia; Chordata; Craniata; Gnathostomata; Actinopterygii; Beryciformes; Holocentroidei; Holocentridae; Neoniphon; kingdom: Animalia; phylum: Chordata; class: Actinopterygii; order: Beryciformes; family: Holocentridae; taxonRank: species; vernacularName: Pence's Squirrelfish; nomenclaturalCode: ICZN; genus: Neoniphon; specificEpithet: pencei; scientificNameAuthorship: Copus, Pyle \& Earle; waterBody: Pacific Ocean; islandGroup: Cook Islands; island: Rarotonga; country: Cook Islands; countryCode: CK; locality: E side; Matavera; off Charles J. Boyle's house; verbatimLocality: Cook Islands; Rarotonga; E side; Matavera; off Charles J. Boyle's house; verbatimDepth: $115 \mathrm{~m}$; minimumDepthInMeters: 115; maximumDepthInMeters: 115; decimalLatitude: -21.223798; decimalLongitude: -159.728123; geodeticDatum: WGS 84; coordinateUncertaintyInMeters: 300; georeferenceSources: Google Earth; samplingProtocol: Spear; eventDate: 2012-07-02; year: 2012; month: 7; day: 2; habitat: small cave near base of vertical drop-off; individualCount: 1; lifeStage: adult; preparations: 55\% Isopropyl; catalogNumber: 237596; recordedBy: David F. Pence; disposition: in collection; associatedSequences: GenBank KJ201926 (Cyt b); KJ188437 (COI); identifiedBy: Richard L. Pyle; dateldentified: 2012-08-07; modified: 2014-10-09T23:30:00Z; language: en; collectionID: urn:Isid:biocol.org:col:1003; institutionCode: CAS; collectionCode: Fish; basisOfRecord: PreservedSpecimen

I. scientificName: Neoniphon pencei; originalNameUsage: Neoniphon pencei Copus, Pyle and Earle, 2014; originalNameUsagelD: 43f5caba-6e4b-42bb-8569-8f93d3502de9; namePublishedln: Copus, Joshua M., Richard L. Pyle \& John L. Earle. 2014. Neoniphon pencei, a new species of holocentrid from Rarotonga, Cook Islands. Biodiversity Data Journal.; namePublishedlnID: bbdce765-389b-4338-9c36-68def122f4fc; nameAccordingTo: Copus, Joshua M., Richard L. Pyle \& John L. Earle. 2014. Neoniphon pencei, a new species of holocentrid from Rarotonga, Cook Islands. Biodiversity Data Journal.; nameAccording ToID: bbdce765-389b-4338-9c36-68def122f4fc; acceptedNameUsage: Neoniphon pencei Copus, Pyle and Earle, 2014; acceptedNameUsage ID: 43f5caba-6e4b-42bb-8569-8f93d3502de9; taxonID: 43f5caba-6 e4b-42bb-8569-8f93d3502de9; scientificNamelD: 43f5caba-6e4b-42bb-8569-8f93d3502d e9; parentNameUsagelD: b047f156-f8da-4ec6-9f64-87345b68a759; parentNameUsage: Neoniphon Castelnau, 1875; higherClassification: Animalia; Deuterostomia; Chordata; Craniata; Gnathostomata; Actinopterygii; Beryciformes; Holocentroidei; Holocentridae; 
Neoniphon; kingdom: Animalia; phylum: Chordata; class: Actinopterygii; order: Beryciformes; family: Holocentridae; taxonRank: species; vernacularName: Pence's Squirrelfish; nomenclaturalCode: ICZN; genus: Neoniphon; specificEpithet: pencei; scientificNameAuthorship: Copus, Pyle \& Earle; waterBody: Pacific Ocean; islandGroup: Cook Islands; island: Rarotonga; country: Cook Islands; countryCode: CK; locality: N side; off Avarua Harbor; verbatimLocality: Cook Islands; Rarotonga; N side; off Avarua Harbor; verbatimDepth: 90 m; minimumDepthInMeters: 90; maximumDepthInMeters: 90; decimalLatitude: -21.198947; decimalLongitude: -159.781353; geodeticDatum: WGS 84; coordinateUncertaintyInMeters: 300; georeferenceSources: Google Earth; samplingProtocol: Quinaldine; eventDate: 2012-06-22; year: 2012; month: 6; day: 22; individualCount: 1; lifeStage: adult; preparations: 55\% Isopropyl; catalogNumber: 431482; recordedBy: John L. Earle; disposition: in collection; otherCatalogNumbers: Formerly BPBM 41195; associatedSequences: GenBank KJ201926; identifiedBy: Richard L. Pyle; dateldentified: 2012-08-07; modified: 2014-10-09T23:30:00Z; language: en; collectionID: urn:Isid:biocol.org:col:1002; institutionCode: USNM; collectionCode: Fish; basisOfRecord: PreservedSpecimen

\section{Other materials:}

a. $\quad$ scientificName: Neoniphon sammara; acceptedNameUsage: N. sammara (Forsskål 1775); parentNameUsage: Neoniphon Castelnau, 1875; higherClassification: Animalia; Deuterostomia; Chordata; Craniata; Gnathostomata; Actinopterygii; Beryciformes; Holocentroidei; Holocentridae; Neoniphon; kingdom: Animalia; phylum: Chordata; class: Actinopterygii; order: Beryciformes; family: Holocentridae; taxonRank: species; vernacularName: Sammara squirelfish; nomenclaturalCode: ICZN; genus: Neoniphon; waterBody: Indian Ocean; islandGroup: Diego Garcia; country: British Indian Ocean Territory; countryCode: IOT; verbatimLocality: Diego Garcia; samplingProtocol: Spear; year: 2002-2011; individualID: NSA128; individualCount: 1; lifeStage: adult; preparations: DMSO; recordedBy: Matt Craig; disposition: in collection; associatedSequences: Genbank-KJ188433; identifiedBy: Matt Craig; language: en

b. scientificName: Neoniphon sammara; acceptedNameUsage: N. sammara (Forsskål 1775); parentNameUsage: Neoniphon Castelnau, 1875; higherClassification: Animalia; Deuterostomia; Chordata; Craniata; Gnathostomata; Actinopterygii; Beryciformes; Holocentroidei; Holocentridae; Neoniphon; kingdom: Animalia; phylum: Chordata; class: Actinopterygii; order: Beryciformes; family: Holocentridae; taxonRank: species; vernacularName: Sammara squirelfish; nomenclaturalCode: ICZN; genus: Neoniphon; waterBody: Indian Ocean; islandGroup: Diego Garcia; country: British Indian Ocean Territory; countryCode: IOT; verbatimLocality: Diego Garcia; samplingProtocol: Spear; year: 2002-2011; individuallD: NSA129; individualCount: 1; lifeStage: adult; preparations: DMSO; recordedBy: Matt Craig; disposition: in collection; associatedSequences: Genbank-KJ188434; identifiedBy: Matt Craig; language: en

c. ScientificName: Neoniphon sammara; acceptedNameUsage: N. sammara (Forsskål 1775); parentNameUsage: Neoniphon Castelnau, 1875; higherClassification: Animalia; Deuterostomia; Chordata; Craniata; Gnathostomata; Actinopterygii; Beryciformes; Holocentroidei; Holocentridae; Neoniphon; kingdom: Animalia; phylum: Chordata; class: Actinopterygii; order: Beryciformes; family: Holocentridae; taxonRank: species; vernacularName: Sammara squirelfish; nomenclaturalCode: ICZN; genus: Neoniphon; waterBody: Indian Ocean; islandGroup: Diego Garcia; country: British Indian Ocean Territory; countryCode: IOT; verbatimLocality: Diego Garcia; samplingProtocol: Spear; year: 2002-2011; individuallD: NSA130; individualCount: 1; lifeStage: adult; preparations: 
DMSO; recordedBy: Matt Craig; disposition: in collection; associatedSequences: Genbank-KJ188435; identifiedBy: Matt Craig; language: en

d. ScientificName: Neoniphon sammara; acceptedNameUsage: N. sammara (Forsskål 1775); parentNameUsage: Neoniphon Castelnau, 1875; higherClassification: Animalia; Deuterostomia; Chordata; Craniata; Gnathostomata; Actinopterygii; Beryciformes; Holocentroidei; Holocentridae; Neoniphon; kingdom: Animalia; phylum: Chordata; class: Actinopterygii; order: Beryciformes; family: Holocentridae; taxonRank: species; vernacularName: Sammara squirelfish; nomenclaturalCode: ICZN; genus: Neoniphon; waterBody: Indian Ocean; islandGroup: Diego Garcia; country: British Indian Ocean Territory; countryCode: IOT; verbatimLocality: Diego Garcia; samplingProtocol: Spear; year: 2002-2011; individuallD: NSA131; individualCount: 1; lifeStage: adult; preparations: DMSO; recordedBy: Matt Craig; disposition: in collection; associatedSequences: Genbank-KJ188436; identifiedBy: Matt Craig; language: en

e. ScientificName: Neoniphon sammara; acceptedNameUsage: N. sammara (Forsskål 1775); parentNameUsage: Neoniphon Castelnau, 1875; higherClassification: Animalia; Deuterostomia; Chordata; Craniata; Gnathostomata; Actinopterygii; Beryciformes; Holocentroidei; Holocentridae; Neoniphon; kingdom: Animalia; phylum: Chordata; class: Actinopterygii; order: Beryciformes; family: Holocentridae; taxonRank: species; vernacularName: Sammara squirelfish; nomenclaturalCode: ICZN; genus: Neoniphon; waterBody: Indian Ocean; islandGroup: Diego Garcia; country: British Indian Ocean Territory; countryCode: IOT; verbatimLocality: Diego Garcia; samplingProtocol: Spear; year: 2002-2011; individualID: NSA132; individualCount: 1; lifeStage: adult; preparations: DMSO; recordedBy: Matt Craig; disposition: in collection; associatedSequences: Genbank-KJ188434; identifiedBy: Matt Craig; language: en

f. ScientificName: Neoniphon sammara; acceptedNameUsage: N. sammara (Forsskål 1775); parentNameUsage: Neoniphon Castelnau, 1875; higherClassification: Animalia; Deuterostomia; Chordata; Craniata; Gnathostomata; Actinopterygii; Beryciformes; Holocentroidei; Holocentridae; Neoniphon; kingdom: Animalia; phylum: Chordata; class: Actinopterygii; order: Beryciformes; family: Holocentridae; taxonRank: species; vernacularName: Sammara squirelfish; nomenclaturalCode: ICZN; genus: Neoniphon; waterBody: Indian Ocean; islandGroup: Diego Garcia; country: British Indian Ocean Territory; countryCode: IOT; verbatimLocality: Diego Garcia; samplingProtocol: Spear; year: 2002-2011; individuallD: NSA133; individualCount: 1; lifeStage: adult; preparations: DMSO; recordedBy: Matt Craig; disposition: in collection; associatedSequences: Genbank-KJ188433; identifiedBy: Matt Craig; language: en

g. scientificName: Neoniphon aurolineatus; acceptedNameUsage: N. aurolineatus Liénard 1839; parentNameUsage: Neoniphon Castelnau, 1875; higherClassification: Animalia; Deuterostomia; Chordata; Craniata; Gnathostomata; Actinopterygii; Beryciformes; Holocentroidei; Holocentridae; Neoniphon; kingdom: Animalia; phylum: Chordata; class: Actinopterygii; order: Beryciformes; family: Holocentridae; taxonRank: species; vernacularName: Yellowstriped squirelfish; nomenclaturalCode: ICZN; genus: Neoniphon; waterBody: Pacific Ocean; islandGroup: Hawaii; island: Oahu; country: United States; countryCode: USA; verbatimLocality: Hawaii; samplingProtocol: Spear; year: 2012; individualID: NAU1; individualCount: 1; lifeStage: adult; preparations: DMSO; recordedBy: Andrew Gray; disposition: in collection; associatedSequences: Genbank-KJ201925; identifiedBy: Andrew Gray; language: en

h. ScientificName: Neoniphon aurolineatus; acceptedNameUsage: $N$. aurolineatus Liénard 1839; parentNameUsage: Neoniphon Castelnau, 1875; higherClassification: Animalia; Deuterostomia; Chordata; Craniata; Gnathostomata; Actinopterygii; Beryciformes; Holocentroidei; Holocentridae; Neoniphon; kingdom: Animalia; phylum: Chordata; class: 
Actinopterygii; order: Beryciformes; family: Holocentridae; taxonRank: species; vernacularName: Yellowstriped squirelfish; nomenclaturalCode: ICZN; genus: Neoniphon; waterBody: Pacific Ocean; islandGroup: Hawaii; island: Oahu; country: United States; countryCode: USA; verbatimLocality: Hawaii; samplingProtocol: Spear; year: 2012; individualID: NAU2; individualCount: 1; lifeStage: adult; preparations: DMSO; recordedBy: Andrew Gray; disposition: in collection; associatedSequences: Genbank-KJ201923; identifiedBy: Andrew Gray; language: en

i. ScientificName: Neoniphon aurolineatus; acceptedNameUsage: $N$. aurolineatus Liénard 1839; parentNameUsage: Neoniphon Castelnau, 1875; higherClassification: Animalia; Deuterostomia; Chordata; Craniata; Gnathostomata; Actinopterygii; Beryciformes; Holocentroidei; Holocentridae; Neoniphon; kingdom: Animalia; phylum: Chordata; class: Actinopterygii; order: Beryciformes; family: Holocentridae; taxonRank: species; vernacularName: Yellowstriped squirelfish; nomenclaturalCode: ICZN; genus: Neoniphon; waterBody: Pacific Ocean; islandGroup: Hawaii; island: Oahu; country: United States; countryCode: USA; verbatimLocality: Hawaii; samplingProtocol: Spear; year: 2012; individualID: NAU3; individualCount: 1; lifeStage: adult; preparations: DMSO; recordedBy: Andrew Gray; disposition: in collection; associatedSequences: Genbank-KJ201924; identifiedBy: Andrew Gray; language: en

j. $\quad$ scientificName: Neoniphon aurolineatus; acceptedNameUsage: $N$. aurolineatus Liénard 1839; parentNameUsage: Neoniphon Castelnau, 1875; higherClassification: Animalia; Deuterostomia; Chordata; Craniata; Gnathostomata; Actinopterygii; Beryciformes; Holocentroidei; Holocentridae; Neoniphon; kingdom: Animalia; phylum: Chordata; class: Actinopterygii; order: Beryciformes; family: Holocentridae; taxonRank: species; vernacularName: Yellowstriped squirelfish; nomenclaturalCode: ICZN; genus: Neoniphon; waterBody: Pacific Ocean; islandGroup: Hawaii; island: Oahu; country: United States; countryCode: USA; verbatimLocality: Hawaii; samplingProtocol: Spear; year: 2012; individuallD: NAU4; individualCount: 1; lifeStage: adult; preparations: DMSO; recordedBy: Andrew Gray; disposition: in collection; associatedSequences: Genbank-KJ201923; identifiedBy: Andrew Gray; language: en

k. ScientificName: Neoniphon aurolineatus; acceptedNameUsage: N. aurolineatus Liénard 1839; parentNameUsage: Neoniphon Castelnau, 1875; higherClassification: Animalia; Deuterostomia; Chordata; Craniata; Gnathostomata; Actinopterygii; Beryciformes; Holocentroidei; Holocentridae; Neoniphon; kingdom: Animalia; phylum: Chordata; class: Actinopterygii; order: Beryciformes; family: Holocentridae; taxonRank: species; vernacularName: Yellowstriped squirelfish; nomenclaturalCode: ICZN; genus: Neoniphon; waterBody: Pacific Ocean; islandGroup: Hawaii; island: Oahu; country: United States; countryCode: USA; verbatimLocality: Hawaii; samplingProtocol: Spear; year: 2012; individuallD: NAU5; individualCount: 1; lifeStage: adult; preparations: DMSO; recordedBy: Andrew Gray; disposition: in collection; associatedSequences: Genbank-KJ201922; identifiedBy: Andrew Gray; language: en

I. ScientificName: Neoniphon aurolineatus; acceptedNameUsage: $N$. aurolineatus Liénard 1839; parentNameUsage: Neoniphon Castelnau, 1875; higherClassification: Animalia; Deuterostomia; Chordata; Craniata; Gnathostomata; Actinopterygii; Beryciformes; Holocentroidei; Holocentridae; Neoniphon; kingdom: Animalia; phylum: Chordata; class: Actinopterygii; order: Beryciformes; family: Holocentridae; taxonRank: species; vernacularName: Yellowstriped squirelfish; nomenclaturalCode: ICZN; genus: Neoniphon; waterBody: Pacific Ocean; islandGroup: Hawaii; island: Oahu; country: United States; countryCode: USA; verbatimLocality: Hawaii; samplingProtocol: Spear; year: 2012; individualID: NAU6; individualCount: 1; lifeStage: adult; preparations: DMSO; recordedBy: 
Andrew Gray; disposition: in collection; associatedSequences: Genbank-KJ201925; identifiedBy: Andrew Gray; language: en

m. ScientificName: Neoniphon aurolineatus; acceptedNameUsage: N. aurolineatus Liénard 1839; parentNameUsage: Neoniphon Castelnau, 1875; higherClassification: Animalia; Deuterostomia; Chordata; Craniata; Gnathostomata; Actinopterygii; Beryciformes; Holocentroidei; Holocentridae; Neoniphon; kingdom: Animalia; phylum: Chordata; class: Actinopterygii; order: Beryciformes; family: Holocentridae; taxonRank: species; vernacularName: Yellowstriped squirelfish; nomenclaturalCode: ICZN; genus: Neoniphon; waterBody: Pacific Ocean; islandGroup: Hawaii; island: Oahu; country: United States; countryCode: USA; verbatimLocality: Hawaii; samplingProtocol: Spear; year: 2012; individualID: NAU7; individualCount: 1; lifeStage: adult; preparations: DMSO; recordedBy: Andrew Gray; disposition: in collection; associatedSequences: Genbank-KJ201925; identifiedBy: Andrew Gray; language: en

n. ScientificName: Neoniphon opercularis; acceptedNameUsage: Neoniphon opercularis Valenciennes 1831; parentNameUsage: Neoniphon Castelnau, 1875; higherClassification: Animalia; Deuterostomia; Chordata; Craniata; Gnathostomata; Actinopterygii; Beryciformes; Holocentroidei; Holocentridae; Neoniphon; kingdom: Animalia; phylum: Chordata; class: Actinopterygii; order: Beryciformes; family: Holocentridae; taxonRank: species; vernacularName: Blackfin squirelfish; nomenclaturalCode: ICZN; genus: Neoniphon; waterBody: Pacific Ocean; islandGroup: Society; island: Moorea; country: French Polynesia; countryCode: PYF; verbatimLocality: Society; samplingProtocol: Spear; year: 2002-2011; individualID: NOP1; individualCount: 1; lifeStage: adult; preparations: DMSO; recordedBy: Matt Craig; disposition: in collection; associatedSequences: Genbank-KJ188432; identifiedBy: Matt Craig; language: en

o. ScientificName: Neoniphon opercularis; acceptedNameUsage: Neoniphon opercularis Valenciennes 1831; parentNameUsage: Neoniphon Castelnau, 1875; higherClassification: Animalia; Deuterostomia; Chordata; Craniata; Gnathostomata; Actinopterygii; Beryciformes; Holocentroidei; Holocentridae; Neoniphon; kingdom: Animalia; phylum: Chordata; class: Actinopterygii; order: Beryciformes; family: Holocentridae; taxonRank: species; vernacularName: Blackfin squirelfish; nomenclaturalCode: ICZN; genus: Neoniphon; waterBody: Pacific Ocean; islandGroup: Society; island: Moorea; country: French Polynesia; countryCode: PYF; verbatimLocality: Society; samplingProtocol: Spear; year: 2002-2011; individuallD: NOP2; individualCount: 1; lifeStage: adult; preparations: DMSO; recordedBy: Matt Craig; disposition: in collection; associatedSequences: Genbank-KJ188432; identifiedBy: Matt Craig; language: en

p. ScientificName: Neoniphon argenteus; acceptedNameUsage: Neoniphon argenteus Valenciennes 1831; parentNameUsage: Neoniphon Castelnau, 1875; higherClassification: Animalia; Deuterostomia; Chordata; Craniata; Gnathostomata; Actinopterygii; Beryciformes; Holocentroidei; Holocentridae; Neoniphon; kingdom: Animalia; phylum: Chordata; class: Actinopterygii; order: Beryciformes; family: Holocentridae; taxonRank: species; vernacularName: Clearfin squirelfish; nomenclaturalCode: ICZN; genus: Neoniphon; waterBody: Pacific Ocean; islandGroup: Line; island: Kiritimati; country: Republic of Kiritimati; countryCode: KIR; verbatimLocality: Line; samplingProtocol: Spear; year: 2002-2011; individualID: NAR1; individualCount: 1; lifeStage: adult; preparations: DMSO; recordedBy: Matt Craig; disposition: in collection; associatedSequences: Genbank-KJ188431; identifiedBy: Matt Craig; language: en

q. $\quad$ scientificName: Neoniphon argenteus; acceptedNameUsage: Neoniphon argenteus Valenciennes 1831; parentNameUsage: Neoniphon Castelnau, 1875; higherClassification: Animalia; Deuterostomia; Chordata; Craniata; Gnathostomata; Actinopterygii; Beryciformes; Holocentroidei; Holocentridae; Neoniphon; kingdom: 
Animalia; phylum: Chordata; class: Actinopterygii; order: Beryciformes; family: Holocentridae; taxonRank: species; vernacularName: Clearfin squirelfish; nomenclaturalCode: ICZN; genus: Neoniphon; waterBody: Pacific Ocean; islandGroup: Line; island: Kiritimati; country: Republic of Kiritimati; countryCode: KIR; verbatimLocality: Line; samplingProtocol: Spear; year: 2002-2011; individualID: NAR2; individualCount: 1; lifeStage: adult; preparations: DMSO; recordedBy: Matt Craig; disposition: in collection; associatedSequences: Genbank-KJ188431; identifiedBy: Matt Craig; language: en

r. scientificName: Neoniphon argenteus; acceptedNameUsage: Neoniphon argenteus Valenciennes 1831; parentNameUsage: Neoniphon Castelnau, 1875; higherClassification: Animalia; Deuterostomia; Chordata; Craniata; Gnathostomata; Actinopterygii; Beryciformes; Holocentroidei; Holocentridae; Neoniphon; kingdom: Animalia; phylum: Chordata; class: Actinopterygii; order: Beryciformes; family: Holocentridae; taxonRank: species; vernacularName: Clearfin squirelfish; nomenclaturalCode: ICZN; genus: Neoniphon; waterBody: Pacific Ocean; islandGroup: Line; island: Kiritimati; country: Republic of Kiritimati; countryCode: KIR; verbatimLocality: Line; samplingProtocol: Spear; year: 2002-2011; individualID: NAR3; individualCount: 1; lifeStage: adult; preparations: DMSO; recordedBy: Matt Craig; disposition: in collection; associatedSequences: Genbank-KJ188431; identifiedBy: Matt Craig; language: en

s. ScientificName: Neoniphon argenteus; acceptedNameUsage: Neoniphon argenteus Valenciennes 1831; parentNameUsage: Neoniphon Castelnau, 1875; higherClassification: Animalia; Deuterostomia; Chordata; Craniata; Gnathostomata; Actinopterygii; Beryciformes; Holocentroidei; Holocentridae; Neoniphon; kingdom: Animalia; phylum: Chordata; class: Actinopterygii; order: Beryciformes; family: Holocentridae; taxonRank: species; vernacularName: Clearfin squirelfish; nomenclaturalCode: ICZN; genus: Neoniphon; waterBody: Pacific Ocean; islandGroup: Line; island: Kiritimati; country: Republic of Kiritimati; countryCode: KIR; verbatimLocality: Line; samplingProtocol: Spear; year: 2002-2011; individualID: NAR4; individualCount: 1; lifeStage: adult; preparations: DMSO; recordedBy: Matt Craig; disposition: in collection; associatedSequences: Genbank-KJ188431; identifiedBy: Matt Craig; language: en

t. scientificName: Neoniphon argenteus; acceptedNameUsage: Neoniphon argenteus Valenciennes 1831; parentNameUsage: Neoniphon Castelnau, 1875; higherClassification: Animalia; Deuterostomia; Chordata; Craniata; Gnathostomata; Actinopterygii; Beryciformes; Holocentroidei; Holocentridae; Neoniphon; kingdom: Animalia; phylum: Chordata; class: Actinopterygii; order: Beryciformes; family: Holocentridae; taxonRank: species; vernacularName: Clearfin squirelfish; nomenclaturalCode: ICZN; genus: Neoniphon; waterBody: Pacific Ocean; islandGroup: Line; island: Kiritimati; country: Republic of Kiritimati; countryCode: KIR; verbatimLocality: Line; samplingProtocol: Spear; year: 2002-2011; individualID: NAR5; individualCount: 1; lifeStage: adult; preparations: DMSO; recordedBy: Matt Craig; disposition: in collection; associatedSequences: Genbank-KJ188431; identifiedBy: Matt Craig; language: en

u. scientificName: Neoniphon argenteus; acceptedNameUsage: Neoniphon argenteus Valenciennes 1831; parentNameUsage: Neoniphon Castelnau, 1875; higherClassification: Animalia; Deuterostomia; Chordata; Craniata; Gnathostomata; Actinopterygii; Beryciformes; Holocentroidei; Holocentridae; Neoniphon; kingdom: Animalia; phylum: Chordata; class: Actinopterygii; order: Beryciformes; family: Holocentridae; taxonRank: species; vernacularName: Clearfin squirelfish; nomenclaturalCode: ICZN; genus: Neoniphon; waterBody: Pacific Ocean; islandGroup: Line; island: Kiritimati; country: Republic of Kiritimati; countryCode: KIR; verbatimLocality: Line; samplingProtocol: Spear; year: 2002-2011; individualID: NAR6; individualCount: 1; 
lifeStage: adult; preparations: DMSO; recordedBy: Matt Craig; disposition: in collection; associatedSequences: Genbank-KJ188431; identifiedBy: Matt Craig; language: en v. ScientificName: Neoniphon marianus; acceptedNameUsage: Neoniphon marianus Cuvier 1829; parentNameUsage: Neoniphon Castelnau, 1875; higherClassification: Animalia; Deuterostomia; Chordata; Craniata; Gnathostomata; Actinopterygii; Beryciformes; Holocentroidei; Holocentridae; Neoniphon; kingdom: Animalia; phylum: Chordata; class: Actinopterygii; order: Beryciformes; family: Holocentridae; taxonRank: species; vernacularName: Longjaw squirelfish; nomenclaturalCode: ICZN; genus: Neoniphon; waterBody: Atlantic Ocean; islandGroup: Bahamas; island: Bahamas; country: Commonwelth of the Bahamas; countryCode: BHS; verbatimLocality: Bahamas; samplingProtocol: Spear; year: 2013; individualID: NMA1; individualCount: 1; lifeStage: adult; preparations: DMSO; recordedBy: Matt Craig; disposition: in collection; associatedSequences: Genbank-KJ201921; identifiedBy: Casey Benkwitt; language: en

\section{Description}

Dorsal rays $\mathrm{XI}, 13$, the last ray branched to base; anal rays IV,9, the last ray branched to base; principal caudal rays 17, the upper and lower unbranched; upper procurrent caudal rays 7 , the first spinous, the last slender and segmented; lower procurrent caudal rays 6 , the first 5 spinous, the last slender and segmented; pectoral rays 14, the uppermost rudimentary, the second and lowermost unbranched; pelvic rays I,7; lateral line scales 49 (48-52); scales above lateral line to base of dorsal spines 5; scales below lateral line to origin of anal fin 6 (6-7); oblique rows of scales on cheek 5 ; vertical row of 9 (8-10) scales on opercle; gill rakers $6+13(6-7+13)$; vertebrae 25 ; body depth 3.2 (2.9-3.3) in SL; head length 2.8 (2.6-2.9) in SL; snout length 3.5 (3.4-3.8) in head length; orbit diameter 3.0 (2.6-3.0) in head length; interorbital width 4.8 (4.1-4.8) in body depth; upper-jaw length 2.3 (2.3-2.6) in head length; preopercular spine 2.2 (2.0-2.9) in orbit diameter; caudal peduncle depth 3.9 (3.7-4.3) in body depth; caudal peduncle length 7.3 (6.5-7.7) in SL, predorsal length 2.7 (2.4-2.7) in SL; preanal length 1.3 (1.2-1.3) in SL; prepelvic length 2.4 (2.4-2.6) in SL; first dorsal spine 3.5 (3.3-4.1) in head length; third dorsal spine longest, 2.3 (2.0-2.8) in head length; first anal spine 29.5 (19.0-29.5) in head length; second anal spine 9.0 (7.2-9.0) in head length; third anal spine 1.3 (1.1-1.3) in head length; fourth anal spine 2.0 (1.7-2.0) in head length; longest anal ray 2.0 (1.9-2.2) in head length; caudal-fin length 5.0 (3.3-5.2) in SL; caudal concavity 2.9 (2.0-2.9) in head length; pectoral-fin length 4.1 (3.6-4.2) in SL; pelvic-spine length 2.3 (2.2-2.5) in head length; pelvic-fin length 4.9 (4.3-4.9) in SL. 
Color in life (Figs 1, 2, 3): Body silvery white with an orange-red tint above lateral line. Scales above lateral line with orange-red borders. Approximately eleven red to orangered stripes following and sometimes bisecting scales of each horizontal scale row, width of stripes on body alternating between very narrow stripes and stripes over three times wider, except for two consecutive wide stripes, numbers six and seven counted ventrally from the thin dorsal-most stripe, the eleventh ventral-most stripe thin and barely visible on some specimens. Preopercle silvery white with a narrow orange-red posterior border, faint on some specimens. Opercle, nape and interorbital space orange-red. Prominent red bar of less than pupil width extending across nape to level of pectoral axil when viewed underwater. Pectoral axil orange-red. Dorsal fin spines and rays light orange red. Membranes of spinous portion of dorsal fin red with white tips and a white semicircular spot encompassing the middle vertical third of each membrane, its greatest length along the preceding anterior spine and not extending to the posterior spine. Some specimens without a white spot on the first membrane. Soft dorsal fin, pectoral fin, anal fin and pelvic fins with transparent membranes, except anal fin with translucent white membrane between longest spines. Pectoral fin and pelvic fin rays with faint pinkish tint. Anal fin spines white with a faint orange tint on some specimens. Anal fin rays orange-red. Caudal fin rays orange-red, faint on inner rays, membranes translucent white.

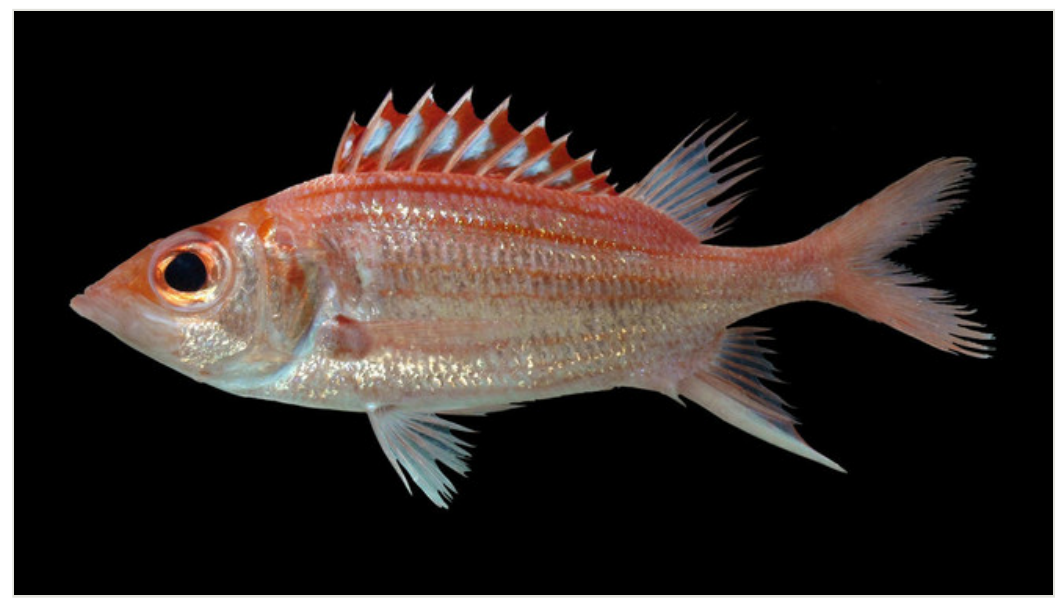

Figure 1.

Holotype of Neoniphon pencei, BPBM 41197, Rarotonga, Cook Islands. Photo: Richard Pyle and Brian Greene.

Color in alcohol: Body pale yellowish-white. Narrow orange-tan stripes bisecting scales of horizontal scale rows, except for lateral line scale row, the stripes above lateral line faint, barely visible on some specimens. Preopercle white with narrow yellow-tan border. Opercle, nape and interorbital space yellow-tan. Spinous dorsal fin membranes translucent with a white tint. Soft dorsal fin, anal fin pectoral fin and pelvic fin membranes transparent. Caudal fin with orange-tan blotch on upper and lower base, 
extending faintly on to upper and lower rays. Middle third of caudal fin rays and membranes transparent.

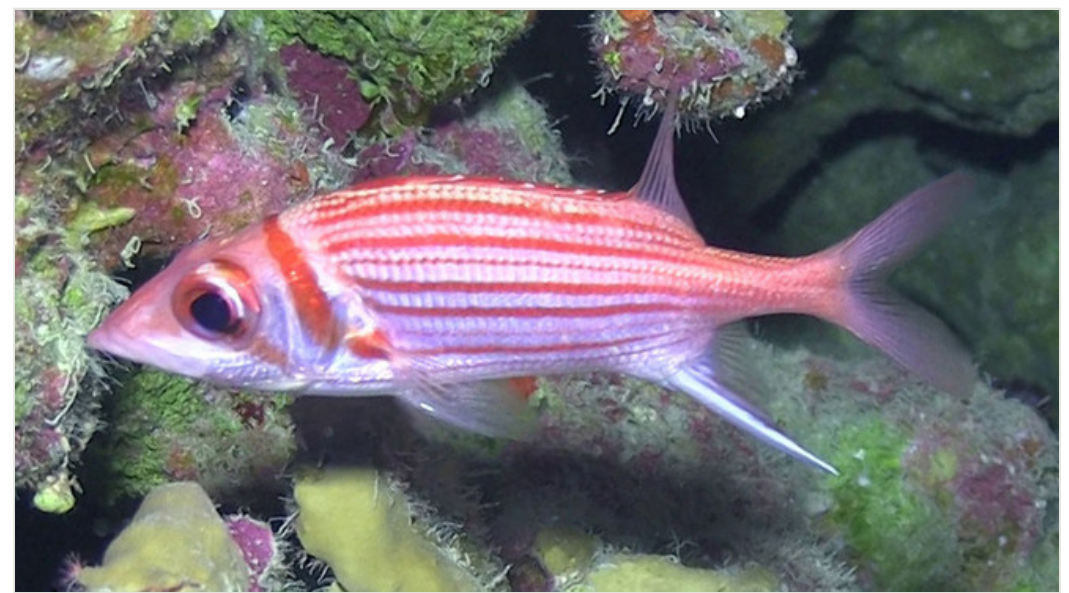

Figure 2.

Neoniphon pencei at approximately $70 \mathrm{~m}$ in Rarotonga, Cook Islands. Cropped from a video frame taken by J.L. Earle.

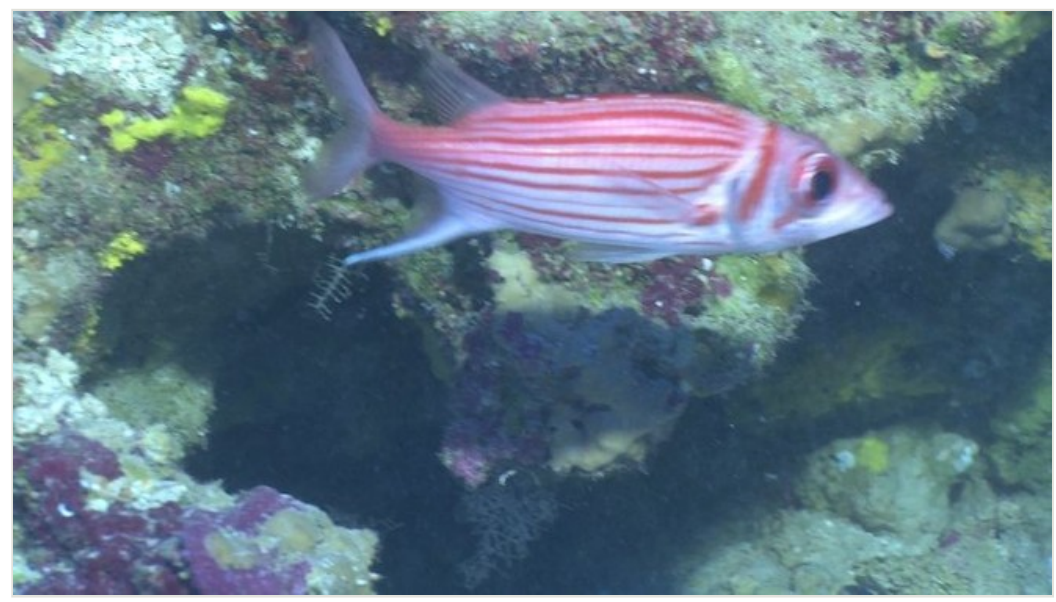

Figure 3.

An apparent Neoniphon pencei at approximately $90 \mathrm{~m}$ in Moorea. Cropped from a video frame taken by R.K. Whitton.

\section{Diagnosis}

Dorsal rays XI,13; anal rays IV,9; pectoral rays 14; lateral-line scales 48-52 (usually 49); scales above lateral line to base of dorsal spines 5 ; scales below lateral line to base of anal fin 6-7; oblique rows of scales on cheek 5; gill rakers $6-7+13$ (usually $6+13$ ); body 
slender, the depth 2.9-3.3 in SL; head length 2.6-2.9 in SL; orbit diameter 2.7-3.0 in head length; interorbital width 4.1-4.8 in body depth; upper jaw length 2.3-2.6 in head; lower jaw strongly protruding; preopercular spine 2.0-2.9 of orbit diameter; first dorsal spine 3.3-4.1 in head length; last dorsal spine shortest; third anal spine the longest, its length 1.1-1.3 in head length; body red with white stripes dorsally, front edge of pelvic and anal fins white, white on base of dorsal fin; reaches $24 \mathrm{~cm}$.

\section{Etymology}

Named for David F. Pence, Dive Safety Officer for the University of Hawai'i, a member of the deep diving team that discovered this species, in recognition of his efforts to collect the type specimens.

\section{Distribution}

All type specimens of $N$. pencei were collected at Rarotonga, Cook Islands. An individual Neoniphon closely matching the life colors of $N$. pencei (and different from all other known species) was captured on video by Robert K. Whitton at a depth of $90 \mathrm{~m}$ at Moorea, in February 2012 (Fig. 3). It is likely that the species is more broadly distributed throughout the southeastern tropical Pacific, but has escaped noticed due to insufficient collecting activities at mesophotic depths in this region.

\section{Analysis}

\section{Genetic results}

After alignment and editing, a 377-bp partial sequence of Cyt b was obtained for all thirtyfive Neoniphon samples, resulting in twelve unique haplotypes. All three phylogenetic methods used resulted in congruent tree topologies and are presented as a Maximum Likelihood reconstruction (Fig. 4). Phylogenetic reconstruction recovered strong support for clades corresponding to known Neoniphon species. The species $N$. pencei showed strong clade support (100\% bootstrap support for all three methods) for belonging to a single clade distinct from currently described Neoniphon species. There was not enough signal to resolve the sister relationship between some members within the genus Neoniphon; however, this description is not necessary for the goals of this study. Neoniphon pencei shows 9-12.5\% uncorrected sequence divergence and 34-47 mutations between all other known Neoniphon species and posesses 8 diagnostic sites unique from all other species of Neoniphon within this this region of Cyt $b$. This is consistent with species level sequence divergence found in other fish taxa (Bellwood et al. 2004, Fessler and Westneat 2007, Randall and Rocha 2009, Rocha 2004, Rocha et al. 2008). 


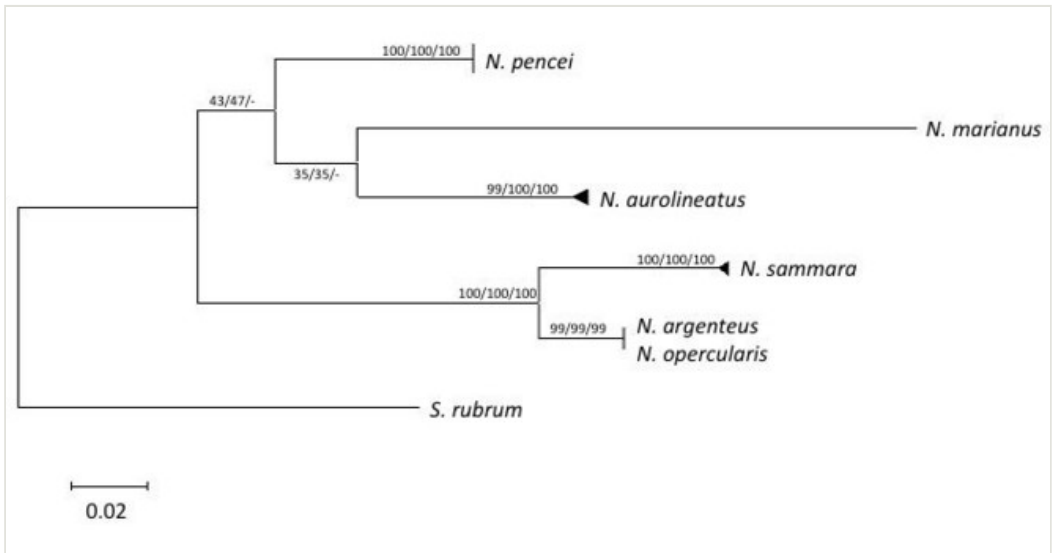

Figure 4.

Maximum likelihood phylogenetic reconstruction for the genus Neoniphon based on Cyt $b$ sequences from 35 individuals, yielding 12 unique haplotypes, rooted with Sargocentron rubrum. Branch support values are Maximum Likelihood, Neighbor-Joining, and Maximum Parsimony bootstrap percent values respectively. Triangles at branch termini represent multiple haplotypes; vertical bars at branch termini represent multiple individuals with identical haplotypes.

\section{Discussion}

Most recent authors who have reported on Neoniphon (e.g., Randall and Heemstra 1985, Randall and Heemstra 1986, Kotlyar 1996, Kotlyar 1998, Randall and Greenfield 1999, Greenfield 2003, Satapoomin 2009) consider it to be a valid genus (a senior synonym of Flammeo), distinct from other genera in the subfamily Holocentrinae (particularly Sargocentron; Fowler 1904), primarily on the basis of the position of the last dorsal-fin spine (relative to the penultimate dorsal-fin spine and first dorsal-fin ray), and the protruding lower jaw in species of Neoniphon (Randall and Heemstra 1985). A more recent phylogenetic analysis of holocentrids by Dornburg et al. (2012), however, reported evidence that Sargocentron and Neoniphon are paraphyletic. Specifically, they found that four of the five species of Neoniphon (they did not include $N$. aurolineatus in their analyses) cluster among several subclades that include nine of the seventeen species of Sargocentron they analyzed ( $S$. coruscum, S. diadema, S. inaequalis, S. ittodai, S. microstoma, S. punctatissimum, S. suborbitalis [=suborbitale], $S$. vexillarium and $S$. xantherythrum). The other eight species of Sargocentron they analyzed ( $S$. caudimaculatum, S. cornutum, S. melanospilos, S. praslin, S. rubrum, S. seychellense, S. spiniferum and $S$. tiere) form a separate clade (their "Sargocentron group 1"). They argue that the characters used to differentiate these species are ecologically plastic and therefore current relationships represent ecotypes rather than their evolutionary relationships. We acknowledge the results of this study and welcome a new comprehensive analysis of the entire Holocentrinae in light of new genetic evidence. However, in the absence of observed 
morphological characters that are consistent with the genetic results, we choose to retain these six species within the genus Neoniphon, to the exclusion of Sargocentron, thereby maintaining nomenclatural stability. Neoniphon pencei clearly differs from all species placed in the genus Sargocentron on the basis of a closer association of the last dorsal-fin spine with the first soft-ray rather than the penultimate spine and the strongly protruding lower jaw (Randall and Heemstra 1985) as well as life color.

Meristic data of the type specimens of Neoniphon pencei are included in Table 1, and proportional measurements are included in Table 2. Neoniphon pencei is distinctive from all other species of holocentrids, both morphologically and genetically. Table 3 summarizes morphological differences between $N$. pencei and other species in the genus. It differs most substantially from all other Neoniphon in number of lateral line scales (48-52, compared with 38-47 among all other species), number of scales above the lateral line to the origin of the dorsal fin (5, compared with 2.5-3.5) and number of scales below the lateral line to the origin of the anal fin (6-7, compared with 7-9). It also differs from $N$. aurolineatus, $N$. opercularis, and $N$. argenteus in proportional length of the upper-jaw (2.3-2.6 in head length, compared with 2.0-2.3), and proportional length of of the third and fourth anal spines (1.1-1.3 and 1.7-2.0, compared with 1.4-1.9 and 1.9-2.7, respectively). It is further distinguished from $N$. aurolineatus in total number of gill rakers (19-20, compared with 15-17); from $N$. opercularis in head length (2.6-2.9 in SL, compared with 2.9-3.1), orbit diameter (1.2-1.4 in head length, compared with 3.0-3.5), snout length (1.2-1.4 in orbit diameter, compared with 1.2-1.5), and interorbital width (1.7-1.9 in orbit diameter, compared with 1.2-1.5); from $N$. argenteus in number of pectoral rays (14, compared with 12-13), interorbital width (1.7-1.9 in orbit diameter, compared with 1.2-1.7), and first dorsalspine length (3.3-4.1 in head length, compared with 2.4-3.1); and from $N$. sammara in number of soft dorsal-fin rays (13, compared with 11-12), interorbital width (1.7-1.9 in orbit diameter, compared with 1.3-1.6), and first dorsal-spine length (3.3-4.1 in head length, compared with 2.2-3.0). In addition to these morphometric characters, $N$. pencei differs from all other species of Neoniphon in life color, particularly in the pattern of white spots on the dorsal fin and overall body color, and the lack of yellow coloration on the body (as in $N$. aurolineatus and $N$. marianus). Genetically, it differs in its Cyt $b$ sequence from $N$. argenteus by $9.8 \%, N$. aurolineatus by $9-9.6 \%, N$. marianus by $11.7 \%, N$. opercularis by $9.8 \%$, and $N$. sammara by $12-12.5 \%$. 
Table 1.

Meristic data of the type specimens of Neoniphon pencei.

\begin{tabular}{|c|c|c|c|c|c|c|c|c|c|c|c|c|c|}
\hline & Holo- & \multicolumn{12}{|c|}{ Paratypes } \\
\hline & $\begin{array}{l}\text { BPBM } \\
41197\end{array}$ & $\begin{array}{l}\text { BPBM } \\
41196\end{array}$ & $\begin{array}{l}\text { BPBM } \\
41196\end{array}$ & $\begin{array}{l}\text { BPBM } \\
41196\end{array}$ & $\begin{array}{l}\text { BPBM } \\
41196\end{array}$ & $\begin{array}{l}\text { BPBM } \\
41196\end{array}$ & $\begin{array}{l}\text { BPBM } \\
41196\end{array}$ & $\begin{array}{l}\text { BPBM } \\
41196\end{array}$ & $\begin{array}{l}\text { BPBM } \\
41196\end{array}$ & $\begin{array}{l}\text { BPBM } \\
41196\end{array}$ & $\begin{array}{l}\text { BPBM } \\
41196\end{array}$ & $\begin{array}{l}\text { USNM } \\
431482\end{array}$ & $\begin{array}{c}\text { CAS } \\
237596\end{array}$ \\
\hline & 175 & 197 & 172 & 170 & 159 & 157 & 162 & 150 & 160 & 160 & 135 & 165 & 132 \\
\hline $\begin{array}{c}\text { Dorsal } \\
\text { Fin } \\
\text { Rays }\end{array}$ & $\mathrm{XI}, 13$ & $\mathrm{XI}, 13$ & $\mathrm{XI}, 13$ & $X I, 13$ & $\mathrm{XI}, 13$ & $\mathrm{XI}, 13$ & $X I, 13$ & $X I, 13$ & $\mathrm{XI}, 13$ & $\mathrm{XI}, 13$ & $\mathrm{XI}, 13$ & $\mathrm{XI}, 13$ & $\mathrm{XI}, 13$ \\
\hline $\begin{array}{l}\text { Anal } \\
\text { Fin } \\
\text { Rays }\end{array}$ & $\mathrm{IV}, 9$ & IV,9 & IV,9 & IV,9 & IV,9 & IV,9 & IV,9 & IV,9 & IV,9 & IV,9 & IV,9 & IV,9 & IV,9 \\
\hline $\begin{array}{c}\text { Pectoral } \\
\text { Fin } \\
\text { Rays }\end{array}$ & 14 & 14 & 14 & 14 & 14 & 14 & 14 & 14 & 14 & 14 & 14 & 14 & 14 \\
\hline $\begin{array}{c}\text { Pelvic } \\
\text { Fin Rays }\end{array}$ & $\mathrm{I}, 7$ & $\mathrm{I}, 7$ & $\mathrm{I}, 7$ & $\mathrm{I}, 7$ & $\mathrm{I}, 7$ & $\mathrm{I}, 7$ & $\mathrm{I}, 7$ & $\mathrm{I}, 7$ & $\mathrm{I}, 7$ & $\mathrm{I}, 7$ & $\mathrm{I}, 7$ & $\mathrm{I}, 7$ & $\mathrm{I}, 7$ \\
\hline $\begin{array}{c}\text { Principal } \\
\text { Caudal } \\
\text { Rays }\end{array}$ & $9+8$ & $9+8$ & $9+8$ & $9+8$ & $9+8$ & $9+8$ & $9+8$ & $9+8$ & $9+8$ & $\begin{array}{c}\text { dama- } \\
\text { ged }\end{array}$ & $9+8$ & $9+8$ & $9+8$ \\
\hline $\begin{array}{l}\text { Upper } \\
\text { Pro- } \\
\text { current } \\
\text { Caudal } \\
\text { Rays }\end{array}$ & 7 & 7 & 7 & 7 & 7 & 7 & 7 & 7 & 7 & $\begin{array}{l}\text { dama- } \\
\text { ged }\end{array}$ & 7 & 7 & 7 \\
\hline $\begin{array}{l}\text { Lower } \\
\text { Pro- } \\
\text { current } \\
\text { Caudal } \\
\text { Rays }\end{array}$ & 6 & 6 & 6 & 6 & 6 & 6 & 6 & 6 & 6 & $\begin{array}{c}\text { dama- } \\
\text { ged }\end{array}$ & 6 & 6 & 6 \\
\hline $\begin{array}{c}\text { Lateral } \\
\text { Line } \\
\text { Scales }\end{array}$ & 49 & 49 & 49 & 49 & 49 & 49 & 49 & 48 & 49 & $\begin{array}{l}\text { dama- } \\
\text { ged }\end{array}$ & 49 & 52 & 49 \\
\hline $\begin{array}{c}\text { Scales } \\
\text { Above } \\
\text { Lateral } \\
\text { Line }\end{array}$ & 5 & 5 & 5 & 5 & 5 & 5 & 5 & 5 & 5 & 5 & 5 & 5 & 5 \\
\hline $\begin{array}{c}\text { Scales } \\
\text { Below } \\
\text { Lateral } \\
\text { Line }\end{array}$ & 7 & 7 & 6 & 7 & 6 & 6 & 6 & 6 & 6 & 6 & 6 & 6 & 7 \\
\hline
\end{tabular}




\begin{tabular}{|c|c|c|c|c|c|c|c|c|c|c|c|c|c|}
\hline $\begin{array}{l}\text { Cheek } \\
\text { Scales }\end{array}$ & 5 & 5 & 5 & 5 & 5 & 5 & 5 & 5 & 5 & 5 & 5 & 5 & 5 \\
\hline $\begin{array}{l}\text { Opercle } \\
\text { Scales }\end{array}$ & 9 & 9 & 10 & 10 & 9 & 10 & 8 & 9 & 10 & 10 & 9 & 9 & 9 \\
\hline $\begin{array}{c}\text { Gill } \\
\text { Rakers }\end{array}$ & $6+13$ & $7+13$ & $6+13$ & $6+13$ & $6+13$ & $6+13$ & $6+13$ & $6+13$ & $7+13$ & $6+13$ & $7+13$ & $6+13$ & $6+13$ \\
\hline $\begin{array}{l}\text { Verte- } \\
\text { brate }\end{array}$ & 25 & 25 & 25 & 25 & 25 & 25 & 25 & 25 & 25 & 25 & 25 & 25 & 25 \\
\hline
\end{tabular}

Table 2.

Proportional measurements of type specimens of Neoniphon pencei as percentages of standard length ${ }^{1}$, head length ${ }^{2}$, orbit diameter ${ }^{3}$, or body depth ${ }^{4}$.

\begin{tabular}{|c|c|c|c|c|c|c|c|c|c|c|c|c|c|}
\hline & Holo- & \multicolumn{12}{|c|}{ Paratypes } \\
\hline & $\begin{array}{l}\text { BPBM } \\
41197\end{array}$ & $\begin{array}{l}\text { BPBM } \\
41196\end{array}$ & $\begin{array}{l}\text { BPBM } \\
41196\end{array}$ & $\begin{array}{l}\text { BPBM } \\
41196\end{array}$ & $\begin{array}{l}\text { BPBM } \\
41196\end{array}$ & $\begin{array}{l}\text { BPBM } \\
41196\end{array}$ & $\begin{array}{l}\text { BPBM } \\
41196\end{array}$ & $\begin{array}{l}\text { BPBM } \\
41196\end{array}$ & $\begin{array}{l}\text { BPBM } \\
41196\end{array}$ & $\begin{array}{l}\text { BPBM } \\
41196\end{array}$ & $\begin{array}{l}\text { BPBM } \\
41196\end{array}$ & $\begin{array}{l}\text { USNM } \\
431482\end{array}$ & $\begin{array}{c}\text { CAS } \\
237596\end{array}$ \\
\hline $\begin{array}{c}\text { Standard } \\
\text { length } \\
(\mathrm{mm})\end{array}$ & 175 & 197 & 172 & 170 & 170 & 157 & 162 & 150 & 160 & 160 & 135 & 165 & 132 \\
\hline $\begin{array}{c}\text { Body } \\
\text { depth }^{1}\end{array}$ & 3.15 & 3.30 & 3.04 & 3.06 & 3.09 & 2.90 & 3.16 & 3.06 & 3.02 & 3.06 & 3.16 & 3.08 & 3.03 \\
\hline $\begin{array}{c}\text { Head } \\
\text { length }^{1}\end{array}$ & 2.82 & 2.93 & 2.77 & 2.79 & 2.83 & 2.60 & 2.72 & 2.69 & 2.74 & 2.70 & 2.81 & 2.84 & 2.73 \\
\hline $\begin{array}{c}\text { Snout } \\
\text { length } 2\end{array}$ & 3.54 & 3.60 & 3.70 & 3.49 & 3.69 & 3.54 & 3.72 & 3.78 & 3.66 & 3.43 & 3.76 & 3.63 & 3.66 \\
\hline $\begin{array}{c}\text { Orbit } \\
\text { diameter }^{2}\end{array}$ & 3.02 & 2.80 & 2.73 & 2.94 & 2.82 & 3.01 & 2.90 & 2.75 & 2.72 & 2.96 & 2.63 & 2.76 & 2.73 \\
\hline $\begin{array}{l}\text { Inter- } \\
\text { orbital } \\
\text { width }^{4}\end{array}$ & 4.83 & 4.69 & 4.11 & 4.72 & 4.78 & 4.70 & 4.18 & 4.45 & 4.51 & 4.35 & 4.38 & 4.46 & 4.61 \\
\hline $\begin{array}{c}\text { Upper- } \\
\text { jaw } \\
\text { length }{ }^{2}\end{array}$ & 2.30 & 2.32 & 2.38 & 2.35 & 2.35 & 2.41 & 2.45 & 2.59 & 2.29 & 2.42 & 2.34 & 2.27 & 2.31 \\
\hline $\begin{array}{c}\text { Preoper- } \\
\text { cular } \\
\text { spine }^{3}\end{array}$ & 2.16 & 2.00 & 2.39 & 2.44 & 2.66 & 2.86 & 2.34 & 2.53 & 1.95 & broken & 2.61 & 2.00 & 2.54 \\
\hline $\begin{array}{l}\text { Caudal- } \\
\text { peduncle } \\
\text { depth }^{4}\end{array}$ & 3.90 & 3.92 & 4.04 & 3.83 & 3.79 & 4.00 & 3.73 & 3.77 & 3.93 & $\begin{array}{c}\text { dama- } \\
\text { ged }\end{array}$ & 4.28 & 4.12 & 4.17 \\
\hline
\end{tabular}




\begin{tabular}{|c|c|c|c|c|c|c|c|c|c|c|c|c|c|}
\hline $\begin{array}{c}\text { Caudal } \\
\text { peduncle } \\
\text { length }^{1}\end{array}$ & 7.30 & 6.74 & 7.24 & 7.23 & 7.16 & 6.82 & 6.48 & 6.45 & 6.81 & $\begin{array}{c}\text { dama- } \\
\text { ged }\end{array}$ & 7.01 & 6.88 & 7.68 \\
\hline $\begin{array}{l}\text { Predorsal } \\
\text { length }^{1}\end{array}$ & 2.66 & 2.72 & 2.52 & 2.64 & 2.54 & 2.43 & 2.58 & 2.53 & 2.43 & 2.46 & 2.53 & 2.61 & 2.52 \\
\hline $\begin{array}{l}\text { Preanal } \\
\text { length } 1\end{array}$ & 1.32 & 1.34 & 1.26 & 1.34 & 1.29 & 1.27 & 1.31 & 1.21 & 1.30 & 1.25 & 1.23 & 1.40 & 1.31 \\
\hline $\begin{array}{l}\text { Prepelvic } \\
\text { length }^{1}\end{array}$ & 2.44 & 2.48 & 2.51 & 2.48 & 2.64 & 2.43 & 2.57 & 2.49 & 2.60 & 2.46 & 2.52 & 2.62 & 2.48 \\
\hline $\begin{array}{c}\text { First } \\
\text { dorsal } \\
\text { spine }^{2}\end{array}$ & 3.50 & 4.14 & 3.99 & broken & 3.66 & 4.09 & 3.65 & 3.54 & 3.86 & 3.65 & 3.45 & 3.84 & 3.33 \\
\hline $\begin{array}{c}\text { Longest } \\
\text { dorsal } \\
\text { spine }^{2}\end{array}$ & 3.34 & 2.44 & 2.30 & 2.37 & 2.31 & 2.37 & 2.25 & 2.03 & 2.40 & 2.68 & 2.24 & 2.85 & 2.21 \\
\hline $\begin{array}{c}\text { First } \\
\text { anal } \\
\text { spine }^{2}\end{array}$ & 29.5 & 25.0 & 25.2 & 23.2 & 27.4 & 23.6 & 23.2 & 25.2 & 26.0 & 22.3 & 24.9 & 25.7 & 19.1 \\
\hline $\begin{array}{l}\text { Second } \\
\text { anal } \\
\text { spine }^{2}\end{array}$ & 9.00 & 8.17 & 8.09 & 7.24 & 8.52 & 8.25 & 8.26 & 7.84 & 8.29 & 7.66 & 7.88 & 7.95 & 7.34 \\
\hline $\begin{array}{l}\text { Third } \\
\text { anal } \\
\text { spine }^{2}\end{array}$ & 1.34 & 1.24 & 1.21 & 1.20 & 1.25 & 1.22 & 1.26 & 1.16 & 1.13 & 1.17 & 1.13 & 1.18 & 1.16 \\
\hline $\begin{array}{c}\text { Fourth } \\
\text { anal } \\
\text { spine }^{2}\end{array}$ & 1.98 & 1.97 & 1.80 & 1.89 & 1.89 & 1.93 & 2.03 & 2.00 & 1.74 & 1.99 & 1.71 & 1.89 & 1.88 \\
\hline $\begin{array}{c}\text { Longest } \\
\text { anal } \\
\text { ray }^{2}\end{array}$ & 2.05 & 2.05 & 1.92 & 1.96 & 2.0 & 2.09 & 2.13 & 2.12 & 1.94 & 2.21 & 1.89 & 2.12 & 1.97 \\
\hline $\begin{array}{l}\text { Caudal- } \\
\text { fin } \\
\text { length }^{1}\end{array}$ & 5.00 & 5.18 & 4.30 & 4.25 & 4.25 & 3.65 & 3.77 & 3.33 & 3.76 & 4.00 & 4.50 & 4.71 & 3.53 \\
\hline $\begin{array}{c}\text { Caudal } \\
\text { concavity }^{2}\end{array}$ & 2.88 & 2.54 & 2.23 & 2.30 & 2.47 & 1.98 & 2.20 & 2.45 & 2.21 & $\begin{array}{l}\text { dama- } \\
\text { ged }\end{array}$ & 2.78 & 2.90 & 2.16 \\
\hline $\begin{array}{l}\text { Pectoral- } \\
\text { fin } \\
\text { length }^{1}\end{array}$ & 4.12 & 4.08 & 4.14 & 4.20 & 4.0 & 3.63 & 3.90 & 3.92 & 4.05 & 3.79 & 3.88 & 4.29 & 4.11 \\
\hline $\begin{array}{c}\text { Pelvic- } \\
\text { spine } \\
\text { length }^{2}\end{array}$ & 2.32 & 2.26 & 2.21 & 2.26 & 2.31 & 2.51 & 2.33 & 2.23 & 2.29 & 2.37 & 2.29 & 2.47 & 2.43 \\
\hline
\end{tabular}




\begin{tabular}{|c|c|c|c|c|c|c|c|c|c|c|c|c|c|c|}
\hline $\begin{array}{c}\text { Pelvic- } \\
\text { fin } \\
\text { length1 }\end{array}$ & 4.93 & 4.83 & 4.74 & 4.42 & 4.59 & 4.30 & 4.63 & 4.41 & 4.44 & 4.54 & 4.58 & 4.93 & 4.57 \\
\hline
\end{tabular}

\section{Table 3.}

Comparison of selected morphological characters for species of Neoniphon. Data for $N$. argenteus, N. aurolineatus (as Flammeo scythrops), N. sammara, and N. opercularis are from Shimizu and Yamakawa (1979); data for N. marianus are from Woods (1955).

Characters that differ from N. pencei are shown in bold. 1 as a proportion of Standard Length; 2 as a a proportion of orbit diameter; 3 as a proportion of head length.

\begin{tabular}{|c|c|c|c|c|c|c|}
\hline Character & N. pencei & $\begin{array}{l}N . \\
\text { argenteus }\end{array}$ & N. aurolineatus & N. marianus & N. opercularis & $\begin{array}{l}\text { N. } \\
\text { sammara }\end{array}$ \\
\hline Head Length ${ }^{1}$ & $2.6-2.9$ & $2.7-3.4$ & $2.8-3.1$ & $2.6-2.9$ & 2.9-3.1 & 2.9-3.2 \\
\hline \multirow[t]{2}{*}{ Snout Length ${ }^{2}$} & $1.2-1.4^{2} \mid$ & $1.2-1.6$ & $1.2-1.5$ & $9.5-10.6^{1}$ & $0.8-1.0$ & $1.1-1.3$ \\
\hline & $9.2-10.6^{1}$ & & & & & \\
\hline \multirow[t]{2}{*}{ Orbit Diameter $^{3}$} & $2.6-3.0^{3}$ & $2.4-3.0$ & $2.5-2.9$ & $6.5-8.2^{1}$ & $3.0-3.5$ & $2.5-3.0$ \\
\hline & $7.4-8.5^{1}$ & & & & & \\
\hline Interorbital & $1.7-1.9^{2} \mid$ & 1.2-1.7 & $1.6-2.1$ & $11.5-14.8^{1}$ & 1.2-1.5 & $1.3-1.6$ \\
\hline Width $^{2}$ & $12.5-15.4^{1}$ & & & & & \\
\hline Upper-jaw & $2.3-2.6^{3} \mid$ & $2.2-2.3$ & $2.0-2.3$ & $5.9-6.7^{1}$ & 2.1-2.2 & $2.2-2.4$ \\
\hline Length $^{3}$ & $6.3-7.0^{1}$ & & & & & \\
\hline First Dorsal- & $3.3-4.1^{3}$ & 2.4-3.1 & $3.2-4.5$ & $14^{1}$ & 3.1.-3.6 & 2.2-3.0 \\
\hline spine Length $^{3}$ & $9.1-12.1^{1}$ & & & & & \\
\hline Third Anal- & $1.1-1.3^{3} \mid$ & $1.4-1.6$ & $1.4-1.6$ & $3.4-4.4^{1}$ & $1.5-1.9$ & $1.1-1.5$ \\
\hline spine Length $^{3}$ & $3.1-3.8^{1}$ & & & & & \\
\hline $\begin{array}{l}\text { Fourth Anal- } \\
\text { spine Length }^{3}\end{array}$ & $1.7-2.0$ & $1.9-2.4$ & 2.1-2.7 & - & $2.0-2.7$ & $1.8-2.2$ \\
\hline $\begin{array}{r}\text { Dorsal-fin soft } \\
\text { rays }\end{array}$ & 13 & $11-13$ & $12-13$ & $12-13$ & 13 & $11-12$ \\
\hline $\begin{array}{r}\text { Pectoral-fin } \\
\text { Rays }\end{array}$ & 14 & $12-13$ & 14 & 14 & $13-14$ & $13-14$ \\
\hline $\begin{array}{r}\text { Lateral-line } \\
\text { Scales }\end{array}$ & $48-52$ & $38-43$ & $44-46$ & $46-47$ & $38-40$ & $39-43$ \\
\hline $\begin{array}{r}\text { Scales Above } \\
\text { Lateral Line }\end{array}$ & 5 & 2.5 & 3.5 & 3.5 & 2.5 & 2.5 \\
\hline $\begin{array}{r}\text { Scales Below } \\
\text { Lateral Line }\end{array}$ & $6-7$ & $7-8$ & $8-9$ & 8 & 8 & 8 \\
\hline
\end{tabular}


Neoniphon pencei appears most similar to $N$. aurolineatus and $N$. marianus, based on having the the fewest number of differences in morphometrics, greatest genetic similarity, and most similar aspects of life coloration with these two species. It is also similar to $N$. aurolineatus in the depth and habitat it occupies. However, the differences between $N$. pencei and these two species as noted above clearly warrant recognition of $N$. pencei as a distinct species. A more comprehensive phylogenetic analysis of the species of Neoniphon and related genera based on both morphology and genetics (with verified voucher specimens) is beyond the scope of this work. 


\section{Acknowledgements}

We are especially grateful to Charles "Chip" Boyle for logistical support in the field, and to David F. Pence for collecting most of the type specimens of the new species. Radiographs were produced by Loreen R. O'Hara, and curatorial support was provided by Arnold Y. Suzumoto. We would also like to thank Casey Benkwitt, Tye Kindinger, Alix Davis, Lillina Tuttle, Mark Hixon, Matthew Craig, Athlene Clarke, Richard Coleman, Brian Bowen, John E. Randall, Brian D. Greene and Robert K. Whitton for their help on various various aspects of this work. We would also like to thank the reviewers for their invaluable comments.

\section{References}

- Baldwin CC, Mounts JH, Smith DG, Weigt LA (2009) Genetic identification and color descriptions of early life-history stages of Belizean Phaeoptyx and Astrapogon (Teleostei: Apogonidae) with comments in identification of adult Phaeoptyx. Zootaxa 2008: 1-22.

- Bellwood DR, van Herwerden L, Konow N (2004) Evolution and biogeography of marine angelfishes (Pisces: Pomacanthidae). Molecular Phylogenetics and Evolution 33: 140-155. DOI: 10.1016/.j.ympev.2004.04.015

- Castelnau FL (1875) Researches on the fishes of Australia. Philadelphia Centennial Expedition of 1876. Intercolonial Exhibition Essays, 1875-6. Intercolonial Exhibitions, Melbourne, 1-52 pp.

- Cuvier G, Valenciennes A (1829) Suite du Livre troisième. Des percoïdes à dorsale unique à sept rayons branchiaux et à dents en velours ou en cardes. Tome troisième. F.G. Levrault, Paris, i-xxviii, [1-2], 1-500, pls. 41-71 pp. [In French].

- Cuvier G, Valenciennes A (1831) Livre septième. Des squamipennes. Livre huitième. Des poissons à pharyngiens labyrinthiformes. Tome septième. F. G. Levrault, Paris, ixxix, 1-531, pls. 170-208 pp. [In French].

- Darriba D, Taboada GL, Doallo R, Posada D (2012) jModelTest 2: more models, new heuristics and parallel computing. Nature Methods 9 (8): 772-772. DOI: $10.1038 /$ nmeth.2109

- Dornburg A, Moore J, Webster R, Warren D, Brandley M, Iglesias T, Wainwright P, Near $\mathrm{T}$ (2012) Molecular phylogenetics of squirrelfishes and soldierfishes (Teleostei: Beryciformes: Holocentridae): Reconciling more than 100 years of taxonomic confusion. Molecular Phylogenetics and Evolution 65 (2): 727-738. [In English]. DOI: 10.1016/ j.ympev.2012.07.020

- Drummond AJ, B. A, Cheung M, Heled J, Kearse M, Moir R, Stones-Havas S, Thierer T, Wilson A (2012) Geneious Pro. 5.6.6. Biomatters. Release date: 201210 15. URL: htt p://www.geneious.com/

- $\quad$ Felsenstein J (1985) Confidence limits on phylogenies: an approach using the bootstrap. Evolution 39: 783-791. DOI: $10.2307 / 2408678$

- Fessler JL, Westneat MW (2007) Molecular phylogenetics of the butterflyfishes (Chaetodontidae): Taxonomy and biogeography of a global coral reef fish family. Molecular Phylogenetics and Evolution 45: 50-68. DOI: 10.1016/j.ympev.2007.05.018 
- Forsskål P (1775) Descriptiones animalium avium, amphibiorum, piscium, insectorum, vermium; quae in itinere orientali observavit Petrus Forskål. Post mortem auctoris edidit Carsten Niebuhr. Adjuncta est materia medica kahirina atque tabula maris Rubri geographica. Mölleri apud Heineck et Faber, Hauniæ [= Copenhagen], 1-20, i-xxxiv, 1-164 pp.

- $\quad$ Fowler HW (1904) New, little known and typical berycoid fishes. Proceedings of the Academy of Natural Sciences of Philadelphia 56: 222-238. [In English].

- $\quad$ Greenfield DW (2003) Holocentridae. In: Carpenter KE (Ed.) The living marine resources of the Western Central Atlantic. Volume 2: Bony fishes part 1 (Acipenseridae to Grammatidae). 2. FAO, Rome, 602-1373 pp. [In English].

- Guindon S, Gascuel O (2003) A simple, fast, and accurate algorithm to estimate large phylogenies by maximum likelihood. Systematic Biology 52: 696-704. [In English]. DOI: $\underline{10.1080 / 10635150390235520}$

- Günther ALG (1859) Catalogue of the acanthopterygian fishes in the collection of the British Museum. Gasterosteidae, Berycidae, Percidae, Aphredoderidae, Pristipomatidae, Mullidae, Sparidae. 1. Taylor \& Francis, London, i-xxxi + 1-524 pp. [In English].

- Jordan D, Evermann B (1903) Descriptions of new genera and species of fishes from the Hawaiian Islands. Bulletin of the United States Fish Commission 22: 161-208. [In English].

- Jordan DS, Evermann BW (1898) The fishes of North and Middle America: a descriptive catalogue of the species of fish-like vertebrates found in the waters of North America north of the Isthmus of Panama. Part III. Bulletin of the United States National Museum 47: 2183a-3136. [In English].

- $\quad$ Kotlyar AN (1996) Beryciform fishes of the world ocean. VNIRO Publishing, 368 pp.

- Kotlyar AN (1998) Species composition and distribution of holocentrids in the oceans of the world (Holocentridae, Beryciformes). Journal of Ichthyology 38 (2): 170-189. [In English].

- Liénard E (1839) Poissons. In: Bouton L (Ed.) Dixième rapport annuel sur les travaux de la Société d'Histoire Naturelle de l'île Maurice. 10. Imprimeire du Cerneen, l'île Maurice.

- Meeker ND, Hutchinson SA, Ho L, Trede NS (2007) Method for isolation of PCR-ready genomic DNA from zebrafish tissues. BioTechniques 43: 610-614. DOI: $\underline{10.2144 / 000112}$ 619

- $\quad$ Pyle R (2000) Assessing undiscovered fish biodiversity on deep coral reefs using advanced self-contained diving technology. Marine Technology Society Journal 34 (4): 82-91. [In English]. DOI: 10.4031/MTSJ.34.4.11

- $\quad$ Randall J (1998) Revision of the Indo-Pacific squirrelfishes (Beryciformes: Holocentridae: Holocentrinae) of the genus Sargocentron, with descriptions of four new species. Indo-Pacific Fishes 28: 1-105. [In English].

- Randall J, Heemstra P (1985) A review of the squirrelfishes of the subfamily Holocentrinae from the western Indian Ocean and Red Sea. Ichthyological Bulletin of the J. L. B. Smith Institute of Ichthyology 49: 1-27.

- $\quad$ Randall JE, Greenfield DW (1999) Family Holocentridae. In: Carpenter KE, Niem VE (Eds) Species identification guide for fisheries purposes. The living marine resources of the western central Pacific. Bony fishes part 2 (Mugilidae to Carangidae). 2. FAO, Rome, 2069-2790 pp. [In English]. 
- $\quad$ Randall JE, Heemstra PC (1986) Holocentrdae. In: Smith MM, Heemstra PC (Eds) Smiths' Sea Fishes. Macmillan South Africa, Johannesburg, 1047 pp. [In English].

- Randall JE, Rocha LA (2009) Chaetodontoplus poliourus, A new angelfish (Perciformes: Pomacanthidae) from the tropical western Atlantic. The Raffles Bulletin of Zoology 57 (2): 511-520.

- Rocha LA (2004) Mitochondrial DNA and color pattern variation in three western Atlantic Halichoeres (Labridae), with the revalidation of two species. Copeia 4: 770-782. DOI: 1 0.1643/CG-04-106

- Rocha LA, Lindeman KC, Rocha CR, Lessios HA (2008) Historical biogeography and speciation in the reef fish genus Haemulon (Teleostei: Haemulidae). Molecular Phylogenetics and Evolution 48: 918-928. DOI: 10.1016/j.ympev.2008.05.024

- $\quad$ Satapoomin U (2009) Holocentridae. In: Kimura S, Satapoomin U, Matsuura K (Eds) Fishes of Andaman Sea, west coast of southern Thailand. National Museum of Nature and Science, Tokyo, $346 \mathrm{pp}$.

- Shimizu T, Yamakawa T (1979) Review of the squirrelfishes (subfamily Holocentrinae: order Beryciformes) of Japan, with a description of a new species. Japanese Journal of Ichthyology 26 (2): 109-147. [In English].

- $\quad$ Song CB, Near TJ, Page JM (1998) Phylogenetic relations among percid fishes as inferred from mitochondrial cytochrome b DNA sequence data. Molecular Phylogenetics and Evolution 10: 343-353. DOI: 10.1006/mpev.1998.0542

- $\quad$ Taberlet P, Meyer A, Bouvet J (1992) Unusually large mitochondrial variation in populations of the blue tit, Parus caeruleus. Molecular Ecology 1: 27-36. [In English]. DOI: $10.1111 / \mathrm{j} .1365-294 X .1992 . t b 00152 . x$

- Tamura K, Peterson D, Peterson N, Stecher G, Nei M, Kumar S (2011) MEGA5: Molecular Evolutionary Genetics Analysis using Maximum Likelihood, Evolutionary Distance, and Maximum Parsimony method. 5.2.2. Molecular Biology and Evolution. URL: www.megasoftware.net

- Woods L (1955) Western Atlantic species of the genus Holocentrus. Fieldiana Zoology 37 (4): 91-119. [In English]. DOI: 10.5962/bhl.title.2972

- Woods L, Sonoda PM (1973) Order Berycomorphi (Beryciformes). Fishes of the western North Atlantic: Halosuriforms, killfishes, squirrelfishes and other beryciforms, stephanoberyciforms, grenadiers. Part 6. The Sears Foundation for Marine Research, Yale University, New Haven, Connecticut, 1-698 pp. [In English]. 